\title{
Modelling New Insecticide-Treated Bed-Nets for Malaria-Vector Control: How to Strategically Manage Resistance?
}

Philip Graham Madgwick

Syngenta Jealott's Hill International Research Centre

Ricardo Kanitz ( $\square$ ricardo.kanitz@syngenta.com )

Syngenta Crop Protection AG https://orcid.org/0000-0003-4759-0207

\section{Research}

Keywords: resistance evolution, modelling, resistance management, vector control, ITNs, insecticides, bednets

Posted Date: July 20th, 2021

DOI: https://doi.org/10.21203/rs.3.rs-721220/v1

License: (c) (1) This work is licensed under a Creative Commons Attribution 4.0 International License. Read Full License

Version of Record: A version of this preprint was published at Malaria Journal on March 24th, 2022. See the published version at https://doi.org/10.1186/s12936-022-04083-z. 


\section{Modelling new insecticide-treated bed-nets for malaria-vector control:}

\section{How to strategically manage resistance?}

3 Philip G. Madgwick ${ }^{1}$, Ricardo Kanitz ${ }^{2 *}$

$4 \quad{ }^{1}$ Syngenta, Jealott's Hill International Research Centre, Bracknell, RG42 6EY, UK.

$5 \quad{ }^{2}$ Syngenta Crop Protection, Rosentalstrasse 67, CH-4058 Basel, Switzerland.

6 * To whom correspondence may be addressed: Ricardo.Kanitz@syngenta.com

7

8 Abstract

9 Background: The programme to eradicate malaria is at a critical juncture as a new wave of insecticides

10 for mosquito control enter their final stages of development. Previous insecticides have been deployed 11 one-at-a-time until their utility was compromised, without the strategic management of resistance. But 12 recent investment has led to the near-synchronous development of new insecticides, and with it the 13 current opportunity to build resistance management into mosquito-control methods to maximize the chance of eradicating malaria.

Methods: Here, building on the parameter framework of an existing mathematical model, resistancemanagement strategies using multiple insecticides are compared to suggest how to deploy combinations of available and new insecticides on bed-nets to achieve maximum impact.

Results: Although results support the use of different strategies in different settings, deploying new insecticides ideally together in (or at least as a part of) a mixture is shown to be a robust strategy across most settings.

Conclusions: Substantially building on previous works, we find alternative solutions for the resistance management of new insecticides to be used in bed-nets for malaria vector control. Our results support a mixture product concept as the most robust way to deploy new insecticides, even if they are mixed with a pyrethroid that has lower effectiveness due to pre-existing resistance. These results can help 
1 deciding on deployment strategies and policies around the sustainable use of these new anti-malaria 2 tools.

3

4 Keywords: resistance evolution; modelling; resistance management; vector control; ITNs; insecticides; 5 bed-nets 


\section{Background}

2 Malaria is a debilitating infectious disease that continues to carry a significant burden on the poorest communities and most vulnerable people around the world; in 2019, 94\% of the estimated 229 million cases of malaria across the world were in Africa and 67\% of the estimated 409,000 deaths occurred in children under 5 years old (World Health Organization, 2020b). Malaria is caused by Plasmodium parasites that have vector-borne transmission between human hosts via mosquitoes. In Africa, $P$.

7 falciparum is the most prevalent parasite accounting for $99.7 \%$ of the cases of malaria in 2018, which

8 is mostly transmitted by Anopheles mosquitoes (especially A. gambiae and A. funestus) (World Health

9 Organization, 2019). Over the last two decades there has been significant investment and progress

10 toward eliminating malaria through vector control; between 2000 and 2015, the international financing

11 of malaria interventions has increased 20 -fold, resulting in a $40 \%$ decrease in the clinical incidence of malaria and averting an estimated 663 million cases of malaria (Bhatt et al., 2015). The greatest contributor accounting for around $68 \%$ of this decrease was the mass distribution of insecticide-treated bed-nets (ITNs) (Bhatt et al., 2015) that both provide a physical barrier that reduces the opportunity for a mosquito to bite a human and an insecticide that kills mosquitoes that attempt to feed on a protected human during their blood-feeding cycle. In contrast to other interventions (of artemisinin-based combination therapy and indoor-residual spray that account for $22 \%$ and $10 \%$ of the decrease, respectively) (Bhatt et al., 2015), ITNs have been successful because they are by far the cheapest intervention and can be mass-distributed freely (or at very low cost) to the poorest communities that tend to be the worst affected by malaria (World Health Organization, 2019). Between 2016 and 2018, over 578 million ITNs were delivered across the world, including to protect at least $40 \%$ of the population in the 10 countries in Africa with the highest prevalence of malaria. In total, since the renewed focus on the eradication of malaria from 2004, over 2 billion ITNs have been delivered worldwide (Roll Back Malaria Partnership, 2020).

There has been growing concern that the increasing levels of resistance may threaten the efficacy of ITNs (and, indeed, other vector control methods of malaria intervention) (Hemingway et al., 2016; 
1 only recommended a single mode of action for use on ITNs - pyrethroids, in part because pyrethroids

2 themselves are inexpensive and so contribute to the low cost manufacture of ITNs (World Health

3 Organization, 2012). Although the clinical incidence of malaria continues to decline year-on-year, the

4 rate of decline is slowing (World Health Organization, 2019) and it is predicted that if pyrethroid-

5 resistance becomes widespread then the trend may reverse, as the cases of malaria averted by ITNs

6 could decrease by more than $40 \%$ (Briët et al., 2013; see also Churcher et al., 2016). This is already

7 beginning to happen in some localities, as the annual decline in the clinical incidence of malaria in the

810 countries in Africa with the highest prevalence of malaria has reversed to an increase in both 2017

9 and 2018 (World Health Organization, 2019). At present, the consensus from numerous studies is that

10 pyrethroid-ITNs remain a more effective vector control method than untreated bed-nets regardless of

11 resistance because mortality tends to be higher even in pyrethroid-resistant strains of mosquitoes

12 (Strode et al., 2014; Kleinschmidt et al., 2018). But results are variable across studies, which may reflect geographical variation in the resistance mechanism affording different levels of resistance. Indeed, across Africa, whilst there has been a dramatic rise in the prevalence of resistance over the last two decades, there is also substantial heterogeneity in the genetics of resistance that differs by region (Strode et al., 2014; Kleinschmidt et al., 2018; Hancock et al., 2020). If malaria interventions continue unchanged and the most effective forms of resistance continue to spread throughout Africa, there is a realistic threat that mosquito control will fail and, in the return of prevalence of malaria pre-2000, clinical incidence and mortality due to malaria would more than double (Hemingway et al., 2016; Yukich \& Chitnis, 2017). Therefore, resistance is a pressing threat to the steps already made toward the eradication of malaria and there is a need for action to be taken now to prevent reversal - let alone any further progress towards eradication.

To-date, the public health approach to combat malaria has been reactive, using the available tools to decrease malaria incidence under a very limited budget that constrains the cost of conceivable interventions (Wilson et al., 2020). In general, little consideration has been given to resistance management (Levick et al., 2017). For example, DDT was used until resistance rendered it ineffective and other insecticides that have subsequently been deployed have had their usage dictated by the balance 
1 of short-term economics and efficacy (Hemingway et al., 2016). However, the threat of insecticide

2 resistance has been proactively responded to before the failure of mosquito control by the Bill and

3 Melinda Gates Foundation in their support of the Innovative Vector Control Consortium (IVCC) with

4 the aim of developing new insecticides for mosquito control in collaboration with industrial partners to

5 eradicate malaria by 2040 . The initial research and development work focused on long-lasting indoor-

6 residual sprays (Innovative Vector Control Consortium, 2020); the first product that was launched into

7 the market in 2013 was Actellic ${ }^{\circledR} 300 \mathrm{CS}$, which was developed in collaboration with Syngenta using

8 the repurposed organophosphate pirimiphos-methyl. The product received full WHO recommendation

9 (World Health Organization, 2013), which was the normative requirement at the time for governments

10 and aid agencies to purchase the product for vector control. A few years later in 2017, the first new

11 long-lasting ITN (Interceptor® G2), which was developed in collaboration with BASF, was launched, combining the existing pyrethroid alpha-cypermethrin and a new pyrazole chlorfenapyr. Whilst three more indoor-residual sprays have been launched in collaboration between the IVCC and Sumitomo and Bayer in recent years, the only other new long-lasting ITN (Royal Guard®) was developed by Disease Control Technologies without assistance from the IVCC and launched in 2019, combining the existing pyrethroid permethrin with the repurposed juvenile hormone analogue pyriproxyfen (that is unusual in primarily affecting mosquito fecundity rather than mortality). These two new long-lasting ITNs have been included on the prequalified list, which is the current normative requirement for governments and aid agencies to purchase them (World Health Organization, 2017, 2020a). Additionally, the IVCC reports that four new chemistries are in the final stages of pre-development with industrial partners (Innovative Vector Control Consortium, 2020) including a novel strobilurin-like insecticide for use on ITNs that is being developed in collaboration with Syngenta - that we will refer to as Syngenta Compound 1 (SC1) (Hueter et al., 2016; Wege et al., 2021). Both Interceptor® G2 and Royal Guard® pair existing and repurposed chemistries, but there is the potential to use combinations of new insecticides like SC1 within a single ITN or across multiple ITNs in a deployment area. With a diversity of new chemistries being developed in relative synchrony, there is the rare opportunity for foresight in integrating resistance management into the design and/or deployment of ITNs to delay the evolution of 
1 resistance to the new insecticides and ensure high levels of mosquito control for a sufficiently long time

2 to provide the greatest chance of eradicating malaria.

3 Here, building on a parameter framework (Levick et al., 2017) that has been extensively analysed

4 (South \& Hastings, 2018; South et al., 2019), conceptual resistance-management strategies using

5 multiple insecticides of rotations, mixtures and mosaics are compared to lower and upper benchmarks

6 to assess what strategy (if any) should be taken to delay the evolution of resistance to the new

7 insecticides. The model setup and analysis differs from Levick et al. (2017) in identified ways,

8 including: (i) the explicit assessment of complementary measures of strategy success such as population

9 control, (ii) the comparison of a broader range of resistance-management strategies and (iii) the

10 examination of a different range of scenarios that include new parameters. Massive simulations with

11 variable parameters are used to manage uncertainties and provide an expectation of why different resistance-management strategies are favoured. As SC1 is the only new insecticide where relevant properties are known to the authors, we give special attention to what strategy should be taken with

14 SC1, which is likely to differ from other new insecticides in having a target-site that is encoded in the mitochondrial genome, as an inhibitor of the cytochrome- $b c l$ complex like azoxystrobin (see Bartlett et al., 2002); this affords it special evolutionary properties that are explicitly treated in our simulations and analysis. Results from the simulations are visualised using conditional inference trees to partition the probabilities of strategy success to describe how a given strategy's success depends on the parameter space. Further, by examination of parts of the parameter space, the potential for different strategies to be best in combination with different partner insecticides (existing pyrethroids $v s$ other new chemistries, based on insecticide effectiveness) and geographic locations (variable zoophily based on female exposure) is considered. In discussion, the practicalities of delivering the recommended strategy are considered to make an initial resistance-management recommendation for SC1 and the other new insecticides.

\section{Methods}

A primary problem for the modelling of resistance evolution is the need to reduce a complex reality into a manageable number of parameters, which also necessarily creates a secondary problem in the 
1 capacity to obtain data to estimate those parameters. These problems are particularly acute when

2 attempting to predict the evolution of resistance to new (i.e. untested) insecticides in mosquitoes. Here

3 we follow the precedent of an existing mathematical model that has been explored using simulations of

4 parameter combinations (Levick et al., 2017), which has provided the parameter framework for other

5 studies as well (South \& Hastings, 2018; South et al., 2019). The model is 'deterministic' in that it only

6 describes the evolutionary pressure from selection, which is suitable for making comparisons about the

7 relative time to resistance under alternative resistance-management strategies because those strategies

8 are primarily focussed on delaying the evolution of resistance by reducing the strength of selection for

9 resistance. The model supposes that there are two insecticides which each have a corresponding

10 resistance locus with a rare resistance allele. Previous analysis has focused on comparing resistance

11 management via mixtures and sequences, where mixtures involve the use of two insecticides until they

12 both fail and sequences involve using one insecticide until it fails before switching to the other until

13 that one fails too. Results show that mixtures tend be favoured when the exposure is low and the

14 effectiveness of insecticides is high. Here, the aim is to compare resistance-management strategies for

$15 \mathrm{SC} 1$ and other new insecticides for mosquito control, which brings with it additional challenges that

16 require modifications to the methods in Levick et al. (2017).

Comparison of methods to Levick et al. (2017)

19 This section does not describe the methods of this paper, but instead describes the key differences

20 between the methods of Levick et al. (2017) and ours. Although this paper uses the same underlying mathematical model of fitness, we have not used the same codebase, which enables the results presented here to act as an independent verification of the results in Levick et al. (2017) using a similar methodology. One of the major differences arises in the choice of parameter space, where Levick et al. (2017) pick the upper and lower limits of parameter ranges for comparison to Curtis (1985), without discussing the choice of range in detail, and randomly sample within the parameter range for a simulation with 10,000 runs. Levick et al. (2017) restrict the parameter range to exclude resistance cost and its dominance term but include a term relating to the correct deployment of mixtures that is ignored 
1 here, which results in a parameter space with 11 parameters. Instead, here, the full range of each

2 parameter is explored across different scenarios using a random sample of 1,000,000 parameter combinations. We have also made a series of additions, alterations and exclusions to the model and approach in Levick et al. (2017) as a result of three key changes that are relevant to the specific questions of this paper, detailed below.

\section{Mitochondrial inheritance}

The simulations in Levick et al. (2017) focus on resistance alleles with nuclear inheritance, albeit that $\mathrm{X}$-chromosomal inheritance is briefly considered but not for analysis using simulations. With special consideration of SC1, there is a need to incorporate an insecticide where target-site resistance evolves at a mitochondrial locus. A resistance allele at a mitochondrial locus is different from a nuclear locus (including the X-chromosome) because of maternal inheritance, (effective) haploidy and the irrelevance of dominance terms. Although SC1 is known to have a target-site that has mitochondrial inheritance, this does not mean that resistance will only ever evolve at the mitochondrial locus as resistance could evolve through mutations that affect regulatory factors or metabolic pathways that would most likely have nuclear inheritance. Further, other new insecticides may not also have a mitochondrially-inherited target-site. As a result, simulations to assess the evolution of resistance to new insecticides are run for combinations of resistance alleles with different modes of inheritance: nuclear inheritance only (nuclear and nuclear; often abbreviated to NN to describe each locus), mixed inheritance (or mitochondrial and nuclear; $\mathrm{MN}$ ) and mitochondrial inheritance only (or mitochondrial and mitochondrial, MM).

Mitochondrial inheritance also impacts the treatment of linkage disequilibrium because it is transmitted between the generations in (what is essentially) the manner of asexual reproduction. In Levick et al. (2017), the differential selection of females and males is permitted to build differential linkage disequilibrium in each sex. Here, this is extended to describe the mitochondrial mode of inheritance (and also make the nuclear mode of inheritance comparable). This adds a substantial degree of complexity to the model because selection must be recorded and accounted in terms of sex-specific haplotypes. Consequently, we also simplify the parameter framework in Levick et al. (2017) to ignore 
1 the 'low concentration insecticide niches' (that can be used to capture the decay of insecticides on bed-

2 nets over their deployment lifespan), which means that here a mosquito is either exposed to a dose of 3 insecticide(s) or it is not.

4 Similarly, in Levick et al. (2017), resistance cost is only applicable to mosquitoes that are not exposed to insecticide, which simplifies the computational process by reducing the dimensions of the parameter space. However, this assumes that resistance cost has a complicated epistatic effect on fitness that is dependent upon the exposure parameters - and hence why resistance cost is dropped in their simulations. Instead, here, resistance cost is attributed irrespective of whether or not a mosquito is exposed to insecticide, which is arguably more biologically realistic. Further, this resistance cost is attributed irrespective of sex (i.e. also to males), which makes the model more complicated but again arguably better reflects biological reality.

\section{Population control}

14 The metric used in Levick et al. (2017) to measure strategy success is the time it takes for a resistance allele to reach $50 \%$ frequency within the population. With two insecticides, this leads to two resulting measures: the time for the first resistance allele to reach 50\% frequency (first-to-break) and the time for the second resistance allele to reach 50\% frequency (second-to-break). This approach is consistent with the majority of genetic models in the literature (REX Consortium, 2010), but is only indirectly related to the goals of resistance management in mosquito control and malaria eradication. For example, as dominance is varied, there is a relevant distinction between resistance allele and phenotype frequencies, as a recessive resistance allele may spread to appreciable frequencies with only a minimal impact on mosquito control. Moreover, deceptively, focusing solely on a genetic assessment of strategy success may inappropriately imply that the optimal resistance-management strategy to delay the time it takes for a resistance allele to reach $50 \%$ frequency would be not to deploy the insecticide at all, but this would not be a very effective method of mosquito control. In reality, the reduction in the population size of mosquitoes is itself only an indirect measure of the ultimate aim to reduce malaria transmission, but there is no obvious conflict between mosquito control and reducing malaria transmission in the way 
that there is between slowing the spread of resistance and mosquito control. Yet, it is worth considering that only female mosquitoes transmit malaria because male mosquitoes do not blood-feed, and so control should reflect the special interest in the female population size. Therefore, a balance needs to be struck between slowing the spread of resistance alleles and the extent of female mosquito control, so both qualities are measured here.

The inclusion of mosquito population size into the parameter framework in Levick et al. (2017) requires the incorporation of a model of mosquito demography. There is a significant challenge in formulating an accurate demographic model that is compatible with the genetic model in Levick et al. (2017). Here, a simple logistic model is used, which can be used to compare strategies but is not calibrated to give an accurate picture of mosquito population change - which is, indeed, also true of the genetic model in Levick et al. (2017). Additionally, an eco-evolutionary approach is adopted, which integrates the change in population size and allele frequencies that would be unchanged in the absence of insecticide. Whilst using the same basic mathematical model, population size is integrated into the model with minimal change. Parents have their population size and allele frequencies modified in relative terms by selection upon interaction with insecticides. Parents may survive between the generations at a rate equal to the complement of the intrinsic death rate. Offspring arise from the random mating of parents, with population size affected by the intrinsic birth rate and density-dependent mortality (that is known to principally affect larvae; Charlwood, 2020). As such, consistent with the simulation framework in Levick et al. (2017), the evolutionary process is modelled in the order of mortality selection on adults from exposure to the insecticide, random mating among surviving adults and offspring production to form a new generation of adults. There is some evidence that most African mosquito species primarily mate before females start their blood-feeding cycle (Takken et al., 2006), which would mean that females acquire sperm before exposure to the insecticide on bed-nets, which could potentially change the strength of selection because the surviving females would have mated before any selection from the insecticide on males (assuming non-zero male exposure). Females mating before blood-feeding is equivalent to zero male exposure, but females mating after blood-feeding is also captured by the framework in Levick et al. (2017) when there is non-zero male exposure. So, herein, it is important to 
1 note that the modelling setup with variable male exposure makes the assumption that males are

2 subjected to selection before mating (and this may not apply to all mosquito species). Therefore, overall,

3 the model of mosquito demography is basic with population size following a standard logistic model

4 whilst allele frequencies follow standard models of population genetics.

5 The logistic model is used to give an equivalent threshold metric for mosquito control, equivalent to

6 how the time it takes for a resistance allele to reach $50 \%$ frequency is a metric for the spread of

7 resistance. Across the scenarios considered, it is important that the chosen threshold is low enough that

8 the female mosquito populations start below it in the first generation that the insecticide is applied, high

9 enough to provide a large quantitative separation between strategies and not-too-high that most female

10 mosquito populations do not reach the threshold within the timeframe that the model is examined across

11 (500 generations). In-keeping with previous work (Gould, 1986), we chose to use the threshold of the time that it takes for the female population to recover to $80 \%$ of its size prior to application of insecticides to balance these considerations. Yet, with the incorporation of demography, there is the possibility of population extinction were the population size to drop below one, which affords a new data type for analysis. It would also be possible to calculate a metric for initial control, but this is used to setup a fairer strategy comparison and so does provide any additional information for the analysis here (see next section).

\section{Strategy comparison}

In Levick et al. (2017), strategy comparison of mixtures and sequences uses the time until resistance in the first- and second-to-break measures. Practically, two insecticides may well be the maximum number of insecticides that can be developed as mixtures on bed-nets due to the requirement of compatible physiochemical properties, but other strategies could easily have more insecticides. However, to make a like-for-like comparison, it seems appropriate to restrict attention to two insecticides. In Levick et al. (2017), mixtures involve the use of two insecticides until they both fail (i.e. 50\% resistance allele frequency); there is some consideration of the incorrect deployment of mixtures (though not with using simulations), which is not examined here. Sequences involve using one insecticide until it fails before 
1 switching to the other until it fails. Necessarily, the setup of strategies introduces a bias in favour of

2 mixtures because a mixture gains the advantage of deployment alongside a partially-effective 3 insecticide (that has $>50 \%$ resistance allele frequency) whereas a sequence never involves the 4 deployment of an insecticide that is partially-effective. Accordingly, the first-to-break is a more 5 comparable measure of strategy success, which is given much more attention in Levick et al. (2017) and also here. A challenge of using this measure, which is not addressed in Levick et al. (2017), is that some of the strategies under comparison (like sequences) involve using insecticides in a particular order, whereas others do not (like mixtures). To avoid introducing a bias and to ensure that a like-for-like comparison is most often made, the insecticide that is first-to-break when solo goes first in the order, which makes the same insecticide most likely to be compared across strategies. This approach becomes particularly relevant when two modes of inheritance are in question (i.e. $\mathrm{MN}$ ).

The biggest difference between the strategy comparison in Levick et al. (2017) and here is in the strategies themselves. Here we examine five resistance-management strategies, albeit that two are treated as 'benchmarks' for the comparison of the other three. As in Levick et al. (2017), sequences provide the default strategy (or, really, non-strategy) of solo deployment where each insecticide is used until it is no longer effective. The insecticide that is the first-to-break is deployed first for comparability (as described in the previous section). Sequences provide a 'lower benchmark' for comparing other strategies in establishing a 'no resistance management' baseline, like a negative control. At the other end of the spectrum, we use the same formulation of the mixture strategy as in Levick et al. (2017) to be an 'upper benchmark' like a positive control. Although dependent upon the dose optimisation process, this strategy is potentially unrealistic because it supposes that the insecticides have the same effectiveness (in terms of mortality) irrespective of whether they are in a mixture or solo. As such, the formulation of the mixture strategy as in Levick et al. (2017) is treated here as the 'maximum' strategy that sets the upper benchmark, which enables the other strategies to be compared within limits of possibility.

The three main strategy concepts under comparison are rotations, mosaics and (our sense of) mixtures. For rotations, insecticides are switched on a fixed term cycle, which corresponds to the three-year 
1 replacement or retreatment schedule of bed-nets of 36 generations. The insecticide that is the first-to-

2 break is deployed first for comparability (as described in the previous section). For mosaics, a mosquito

3 has an exclusive 50\% chance of being exposed to one or other insecticide, which is as if ITNs are

4 equally distributed throughout a population. The relationship between rotations and mosaics can be

5 summarised by initial control, which is calculated as the complement of mean fitness $(1-\bar{\omega}$; see next

6 section for fitness equations). The average initial control from rotations when both insecticides are first

7 deployed is equal to the initial control from mosaics, which sets up as close a like-for-like comparison

8 as possible with rotations only differing from mosaics in the temporal dimension of insecticide

9 deployment. In deviation from their attribution in Levick et al. (2017), mixtures can also be attributed

10 to have the same average initial control as rotations and mosaics to make for a similarly like-for-like

11 comparison. Mixtures differ from mosaics in that if a mosquito is exposed to the ITN, it is inclusively exposed to both insecticides. This can be standardised to ensure the same average initial control by applying a constant to the effectiveness of each insecticide, which reflects the dose optimisation process, which can be thought of as implying rough cost-equivalence (given that the cost of the ITN is largely the cost of the insecticide). Such considerations of economic cost may seem superfluous for a comparison of strategy concepts as in here, but they are essential for making fair comparisons among practical alternatives.

An additional practical constraint of the simulations in Levick et al. (2017) is that the measure for strategy comparison is only recoded if it is within a finite timeframe because of computational constraints. In Levick et al. (2017), the relevant timeframe for a resistance-management strategy was identified as 500 mosquito generations; this roughly corresponds to 40 years (assuming a generation per month), which exceeds the time it would take to develop a new insecticide for use on ITNs. Consequently, the analysis in Levick et al. (2017) excludes all comparisons where at least one strategy leads to a resistance allele not reaching $50 \%$ frequency within 500 generations. Necessarily, this approach excludes comparisons where one strategy is highly successful at delaying the evolution of resistance, which in the comparison in Levick et al. (2017) would bias results against (their sense) mixtures (or, our sense, the maximum benchmark). The extent of the bias is difficult to assess, but it 
1 would potentially start to matter much more when a larger number of strategies are compared because

2 it is more likely for at least one to take more than 500 generations to reach $50 \%$ resistance allele

3 frequency. Therefore, so as not to introduce a bias against comparisons where a particular strategy is very successful, if a strategy does not reach $50 \%$ resistance allele frequency within 500 generations but the resistance allele nonetheless has an increasing frequency, then the output can be given a nominal value above the upper limit to include this outcome in as far as possible in the strategy comparison.

Mathematical description of selection in the model

9 This section provides a technical description of the fitness model consistent with the parameter framework in Levick et al. (2017) but with the additions, alterations and exclusions identified above. The notation for this section is summarised in Table 1. At the start of a simulation of a parameter combination, alleles are assumed to be in linkage equilibrium. The fitness of an individual with particular alleles and sex can be described as a function of model parameters (Table 2, 3), although inkeeping with Levick et al. (2017) it is simpler to describe male individuals with particular alleles as a function of female individuals with those alleles due to the formulation of the male exposure term as a percentage of female exposure (Table 3). For a given generation, the fitness model depends upon how many insecticides are in use and at what rate, which depends upon the strategy - although this really reflects a concept of mosquito interaction with an ITN more so than a practical description of ITN design or deployment:

Sequences are the simplest 'strategy', where one insecticide is $100 \%$ in-use at any one point in time and this strategy switches to the second insecticide once the resistance allele to the first in-use insecticide exceeds $50 \%$ frequency (i.e. switching from insecticide niche: A,- to -,B). This corresponds to the default scenario (or 'non-strategy') where an insecticide is used on an ITN until its efficacy starts to fail and then a new insecticide is used instead. The order in that insecticides are used is determined such that the first-to-break goes first. 
Rotations are similar, except that the in-use insecticide switches every 36 generations, which corresponds to the recommended three-year replacement or retreatment schedule of ITNs (World Health Organization, 2001). The first- and second-to-break measures could be crossed multiple times with fluctuating allele frequencies, whereupon the first crossing of the threshold is always recorded. For the most part, the exact length of the replacement or retreatment schedule is essentially arbitrary insofar as switch-period is much smaller than the time to the threshold of resistance (i.e. 50\% resistance allele frequency), and this is even more true with the 'soft' analysis of the threshold data (see next section). Like sequences, the order of insecticides is with the first-to-break going first.

Mosaics involve both insecticides being 50\% in-use at the same time (i.e. corresponding to insecticide niches: $1 / 2$ A,- and $1 / 2-, B$ ), assuming that a mosquito would only encounter one insecticide within a generation, as if 50\% of ITNs had each of the two insecticides across the population (that, due to practical constraints, may reflect $100 \%$ usage in different deployment areas).

Mixtures involve an exposed mosquito encountering both insecticides at a reduced rate $k$ (i.e. insecticide niche: $k$ A,B), as if both insecticides were on the same ITN. $k$ is a constant for a particular parameter combination that ensures that mixtures have the same average initial control as rotations and mosaics for their like-for-like comparison, which is assumed to reflect the balance of formulating each insecticide for use as a mixture with any corresponding modifications of insecticide dose. This constant is calculated as a function of the effectiveness of the two insecticides $\left(m_{1}, m_{2}\right.$; see Table 1$)$ :

$$
k=\frac{m_{1}+m_{2}-\sqrt{\left(m_{1}+m_{2}\right)^{2}-2 m_{1} m_{2}\left(m_{1}+m_{2}\right)}}{2 m_{1} m_{2}}
$$

'Maximum' describes an upper benchmark (like sequences provide a lower benchmark) for the comparison of strategies that are the same as mixtures but assume that $k=1$, which could be plausible were a mosquito to acquire the same dosage of the two insecticides from one or 


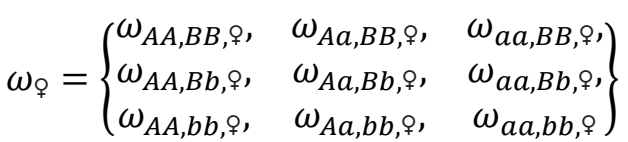

$9 \quad$ And the fitness vector of male individuals:

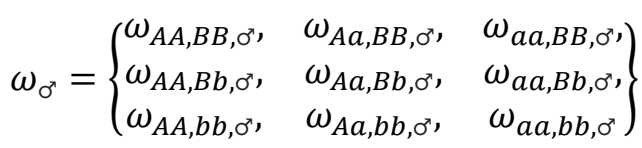

10 And taking their cross-product, which produces a matrix that describes the different frequencies of

11 matings between females and males with different genotypes. Each entry of this matrix describes a

12 distribution of offspring genotypes, and so the frequencies of matings are then decomposed, collecting

13 offspring frequency by both genotype and sex. Modelling breeding in this way permits selection to build

14 linkage disequilibrium with respect to the resistance alleles at the two loci and also with respect to their sex.

16 In the simplest model, offspring frequency by genotype and sex represents the distribution of

17 individuals in the next generation, but the model also considers population size, overlapping generations

18 and density-dependence. Population size and its change is modelled using a simple logistic model using

19 an intrinsic birth rate parameter $(b)$. Overlapping generations are included using an intrinsic death rate

20 parameter $(d)$ that describes the percentage of breeding adults that survive into the next generation. In

21 mosquitoes, density-dependence is primarily a property of breeding sites rather than feeding sites 
1 (Charlwood, 2020), with competition taking place among larvae that are in the next generation.

2 Consequently, density-dependence is attributed to offspring only, which is attributed such that in the

3 absence of the insecticide(s) the population of females and males $\left(N_{\varphi}, N_{\mathrm{O}^{\prime}}\right)$ remains constant at its initial

4 population size ( $N / 2$ for each sex) as the carrying capacity. Accordingly, with respect to the vector of

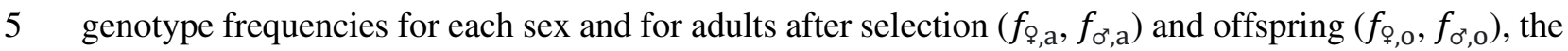

6 vector of the number of females and males with genotypes in the next generation $\left(f_{q_{,},}^{\prime} N_{\varphi_{q}}^{\prime}, f_{\sigma^{\prime}, \mathrm{a}}^{\prime} N_{\mathrm{o}^{\prime}}^{\prime}\right)$ is

7 given as:

$$
\begin{aligned}
& f_{\varphi}^{\prime} N_{\varphi}^{\prime}=N_{\varphi} f_{q, \mathrm{a}} \omega_{\varphi}(1-d)+\left(\sum N_{\varphi} f_{q, \mathrm{a}} \omega_{\varphi}\right) f_{q, \mathrm{o}}\left(1+b-\frac{2(1+b-d)\left(\sum N_{\varphi} f_{q, \mathrm{a}} \omega_{\varphi}\right)}{N}\right) \\
& f_{\sigma^{\prime}}^{\prime} N_{\sigma^{\prime \prime}}^{\prime}=N_{\sigma^{\prime}} f_{\sigma^{\prime \prime}, \mathrm{a}} \omega_{\sigma^{\prime \prime}}(1-d)+\left(\sum N_{\phi} f_{q, \mathrm{a}} \omega_{q}\right) f_{q, \mathrm{o}}\left(1+b-\frac{2(1+b-d)\left(\sum N_{q} f_{q, \mathrm{a}} \omega_{q}\right)}{N}\right)
\end{aligned}
$$

The total number of females in the next generation is simply the sum of the vector of the number of

9 females with genotypes in the next generation $\left(N_{o}^{\prime}=\sum f_{q}^{\prime} N_{q}^{\prime}\right)$ and the same for the total number of

10 males $\left(N_{\sigma^{\prime}}^{\prime}=\sum f_{\sigma^{\prime}}^{\prime} N_{O^{\prime}}^{\prime}\right)$, as the genotype frequency vector necessarily sums to 1 . The vector of genotype

11 frequency by sex can then be decomposed into the total allele frequencies in the population to calculate the measures of strategy success (as appropriate). 
1 Table 1. Parameter framework and model notation.

\begin{tabular}{|c|c|c|c|}
\hline \multicolumn{2}{|l|}{ Parameter } & Definition & Notation \\
\hline \multicolumn{2}{|c|}{ Population size } & $\begin{array}{l}\text { starting population size (and carrying capacity in } \\
\text { logistic model) }\end{array}$ & $N$ \\
\hline \multicolumn{2}{|c|}{ Intrinsic birth rate } & $\%$ population growth rate (in logistic model) & $b$ \\
\hline \multicolumn{2}{|c|}{ Intrinsic death rate } & $\%$ breeding mosquitoes that die into next generation & $d$ \\
\hline \multirow{2}{*}{ Exposure } & Female & $\%$ female mosquitoes that receive a dose & $x_{Q}$ \\
\hline & Male & $\%$ male mosquitoes that receive a dose & $x_{0^{\pi}}$ \\
\hline \multirow{2}{*}{$\begin{array}{l}\text { Insecticide } \\
\text { effectiveness }\end{array}$} & Insecticide 1 & $\%$ dosed mosquitoes that die from insecticide 1 & $m_{1}$ \\
\hline & Insecticide 2 & $\%$ dosed mosquitoes that die from insecticide 2 & $m_{2}$ \\
\hline \multirow{2}{*}{$\begin{array}{l}\text { Initial } \\
\text { frequency }\end{array}$} & Allele A & starting frequency of allele A & $f_{0, A}$ \\
\hline & Allele B & starting frequency of allele B & $f_{0, B}$ \\
\hline \multirow{2}{*}{$\begin{array}{l}\text { Resistance } \\
\text { restoration }\end{array}$} & Allele A & $\%$ return to baseline fitness with resistance allele $\mathrm{A}$ & $r_{A}$ \\
\hline & Allele B & $\%$ return to baseline fitness with resistance allele $\mathrm{B}$ & $r_{B}$ \\
\hline \multirow{2}{*}{$\begin{array}{l}\text { Dominance } \\
\text { of resistance } \\
\text { restoration }\end{array}$} & Allele A & $\%$ resistance restoration in heterozygote with allele $\mathrm{A}$ & $h_{A}^{r}$ \\
\hline & Allele B & $\%$ resistance restoration in heterozygote with allele B & $h_{B}^{r}$ \\
\hline \multirow{2}{*}{$\begin{array}{l}\text { Resistance } \\
\text { cost }\end{array}$} & Allele A & $\%$ non-dosed mosquitoes that die from carrying allele $\mathrm{A}$ & $c_{A}$ \\
\hline & Allele B & $\%$ non-dosed mosquitoes that die from carrying allele $\mathrm{B}$ & $c_{B}$ \\
\hline \multirow{2}{*}{$\begin{array}{l}\text { Dominance } \\
\text { of resistance } \\
\text { cost }\end{array}$} & Allele A & $\%$ resistance cost in heterozygote with allele A & $h_{A}^{c}$ \\
\hline & Allele B & $\%$ resistance cost in heterozygote with allele B & $h_{B}^{c}$ \\
\hline
\end{tabular}


Table 2. Female fitness separated across insecticide niches, where '-' represents no insecticide, ' 1 ' represents insecticide 1 and ' 2 ' represents insecticide 2 . Labelling is

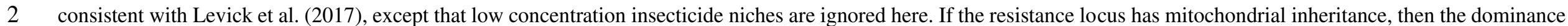
3 terms are dropped.

\begin{tabular}{|c|c|c|c|c|}
\hline Notation &,-- & $1,-$ &,- 2 & 1,2 \\
\hline$\omega_{A A, B B, q}=$ & $\left(1-x_{q}\right)\left(1-c_{A}\right)\left(1-c_{B}\right)$ & $\begin{array}{l}x_{\uparrow}\left(1-m_{1}\left(1-r_{A}\right)\right) \\
\left(1-c_{A}\right)\left(1-c_{B}\right)\end{array}$ & $\begin{array}{l}x_{\uparrow}\left(1-m_{2}\left(1-r_{B}\right)\right) \\
\left(1-c_{A}\right)\left(1-c_{B}\right)\end{array}$ & $\begin{array}{l}x_{\varphi}\left(1-m_{1}\left(1-r_{A}\right)\right) \\
\left(1-m_{2}\left(1-r_{B}\right)\right) \\
\left(1-c_{A}\right)\left(1-c_{B}\right)\end{array}$ \\
\hline$\omega_{A a, B B, \uparrow}=$ & $\left(1-x_{\varphi}\right)\left(1-h_{A}^{c} c_{A}\right)\left(1-c_{B}\right)$ & $\begin{array}{l}x_{\risingdotseq}\left(1-m_{1}\left(1-h_{A}^{r} r_{A}\right)\right) \\
\left(1-h_{A}^{c} c_{A}\right)\left(1-c_{B}\right)\end{array}$ & $\begin{array}{l}x_{q}\left(1-m_{2}\left(1-r_{B}\right)\right) \\
\left(1-h_{A}^{c} c_{A}\right)\left(1-c_{B}\right)\end{array}$ & $\begin{array}{l}x_{\varphi}\left(1-m_{1}\left(1-h_{A}^{r} r_{A}\right)\right) \\
\left(1-m_{2}\left(1-r_{B}\right)\right) \\
\left(1-h_{A}^{c} c_{A}\right)\left(1-c_{B}\right)\end{array}$ \\
\hline$\omega_{a a, B B, q}=$ & $\left(1-x_{\varphi}\right)\left(1-c_{B}\right)$ & $x_{\varphi}\left(1-m_{1}\right)\left(1-c_{B}\right)$ & $\begin{array}{l}x_{\uparrow}\left(1-m_{2}\left(1-h_{B}^{r} r_{B}\right)\right) \\
\left(1-c_{B}\right)\end{array}$ & $\begin{array}{l}x_{q}\left(1-m_{1}\right) \\
\left(1-m_{2}\left(1-h_{B}^{r} r_{B}\right)\right) \\
\left(1-c_{B}\right)\end{array}$ \\
\hline$\omega_{A A, B b, q}=$ & $\left(1-x_{q}\right)\left(1-c_{A}\right)\left(1-h_{B}^{c} c_{B}\right)$ & $\begin{array}{l}x_{\uparrow}\left(1-m_{1}\left(1-r_{A}\right)\right) \\
\left(1-c_{A}\right)\left(1-h_{B}^{c} c_{B}\right)\end{array}$ & $\begin{array}{l}x_{\varphi}\left(1-m_{2}\left(1-h_{B}^{r} r_{B}\right)\right) \\
\left(1-c_{A}\right)\left(1-h_{B}^{c} c_{B}\right)\end{array}$ & $\begin{array}{l}x_{q}\left(1-m_{1}\left(1-r_{A}\right)\right) \\
\left(1-m_{2}\left(1-h_{B}^{r} r_{B}\right)\right) \\
\left(1-c_{A}\right)\left(1-h_{B}^{c} c_{B}\right)\end{array}$ \\
\hline$\omega_{A a, B b, q}=$ & $\left(1-x_{q}\right)\left(1-h_{A}^{c} c_{A}\right)\left(1-h_{B}^{c} c_{B}\right)$ & $\begin{array}{l}x_{\varphi}\left(1-m_{1}\left(1-h_{A}^{r} r_{A}\right)\right) \\
\left(1-h_{A}^{c} c_{A}\right)\left(1-h_{B}^{c} c_{B}\right)\end{array}$ & $\begin{array}{l}x_{\varphi}\left(1-m_{2}\left(1-h_{B}^{r} r_{B}\right)\right) \\
\left(1-h_{A}^{c} c_{A}\right)\left(1-h_{B}^{c} c_{B}\right)\end{array}$ & $\begin{array}{l}x_{\varphi}\left(1-m_{1}\left(1-h_{A}^{r} r_{A}\right)\right) \\
\left(1-m_{2}\left(1-h_{B}^{r} r_{B}\right)\right) \\
\left(1-h_{A}^{c} c_{A}\right)\left(1-h_{B}^{c} c_{B}\right)\end{array}$ \\
\hline$\omega_{a a, B b, q}=$ & $\left(1-x_{q}\right)\left(1-h_{B}^{c} c_{B}\right)$ & $x_{\varphi}\left(1-m_{1}\right)\left(1-h_{B}^{c} c_{B}\right)$ & $\begin{array}{l}x_{\uparrow}\left(1-m_{2}\left(1-h_{B}^{r} r_{B}\right)\right) \\
\left(1-h_{B}^{c} c_{B}\right)\end{array}$ & $\begin{array}{l}x_{\varphi}\left(1-m_{1}\right) \\
\left(1-m_{2}\left(1-h_{B}^{r} r_{B}\right)\right) \\
\left(1-h_{B}^{c} c_{B}\right)\end{array}$ \\
\hline$\omega_{A A, b b, q}=$ & $\left(1-x_{\varphi}\right)\left(1-c_{A}\right)$ & $x_{\odot}\left(1-m_{1}\left(1-r_{A}\right)\right)\left(1-c_{A}\right)$ & $x_{\varphi}\left(1-m_{2}\right)\left(1-c_{A}\right)$ & $\begin{array}{l}x_{q}\left(1-m_{1}\left(1-r_{A}\right)\right) \\
\left(1-m_{2}\right)\left(1-c_{A}\right)\end{array}$ \\
\hline$\omega_{A a, b b, q}=$ & $\left(1-x_{\odot}\right)\left(1-h_{A}^{c} c_{A}\right)$ & $\begin{array}{l}x_{\uparrow}\left(1-m_{1}\left(1-h_{A}^{r} r_{A}\right)\right) \\
\left(1-h_{A}^{c} c_{A}\right)\end{array}$ & $x_{\varphi}\left(1-m_{2}\right)\left(1-h_{A}^{c} c_{A}\right)$ & $\begin{array}{l}x_{\uparrow}\left(1-m_{1}\left(1-h_{A}^{r} r_{A}\right)\right) \\
\left(1-m_{2}\right)\left(1-h_{A}^{c} c_{A}\right)\end{array}$ \\
\hline$\omega_{a a, b b, q}=$ & $\left(1-x_{q}\right)$ & $x_{\varphi}\left(1-m_{1}\right)$ & $x_{\wp}\left(1-m_{2}\right)$ & $x_{\varphi}\left(1-m_{1}\right)\left(1-m_{2}\right)$ \\
\hline
\end{tabular}


1 Table 3. Male fitness as a function of female fitness. If the resistance locus has mitochondrial inheritance, then the dominance terms are dropped.

\begin{tabular}{|c|c|}
\hline Notation & Expression \\
\hline$\omega_{A A, B B, 0^{*}}=$ & $\left(1-x_{\sigma^{\top}}\right)\left(1-c_{A}\right)\left(1-c_{B}\right)+x_{\sigma^{\top} \omega_{A A, B B, q}}$ \\
\hline$\omega_{A a, B B, 0^{\circ}}=$ & $\left(1-x_{\sigma^{\prime}}\right)\left(1-h_{A}^{c} c_{A}\right)\left(1-c_{B}\right)+x_{\sigma^{\prime} \omega_{A a, B B, q}}$ \\
\hline$\omega_{a a, B B, 0^{*}}=$ & $\left(1-x_{\sigma^{\prime}}\right)\left(1-c_{B}\right)+x_{\sigma^{\prime}} \omega_{a a, B B, q}$ \\
\hline$\omega_{A A, B b, 0^{*}}=$ & $\left(1-x_{\Im^{\top}}\right)\left(1-c_{A}\right)\left(1-h_{B}^{r} c_{B}\right)+x_{\sigma^{\top} \omega_{A A, B b, q}}$ \\
\hline$\omega_{A a, B b, 0^{*}}=$ & $\left(1-x_{\sigma^{\prime}}\right)\left(1-h_{A}^{c} c_{A}\right)\left(1-h_{B}^{r} c_{B}\right)+x_{\sigma^{\pi} \omega_{A a, B b, 9}}$ \\
\hline$\omega_{a a, B b, 0^{*}}=$ & $\left(1-x_{\sigma^{\prime}}\right)\left(1-h_{B}^{r} c_{B}\right)+x_{\sigma^{\Im} \omega_{a a, B b, q}}$ \\
\hline$\omega_{A A, b b, 0^{\prime}}=$ & $\left(1-x_{\sigma^{\prime}}\right)\left(1-c_{A}\right)+x_{\sigma^{\prime}} \omega_{A A, b b, q}$ \\
\hline$\omega_{A a, b b, 0^{0^{*}}}=$ & $\left(1-x_{\sigma^{\Im}}\right)\left(1-h_{A}^{c} c_{A}\right)+x_{\mho^{\curvearrowright} \omega_{A a, b b, q}}$ \\
\hline$\omega_{a a, b b, 0^{*}}=$ & $\left(1-x_{\sigma^{\top}}\right)+x_{\sigma^{\pi} \omega_{a a, b b, q}}$ \\
\hline
\end{tabular}


2 The full range of each of the 17 parameters of the genetic model of resistance evolution is explored in 3 a parameter space using the same random sample of 1,000,000 parameter combinations for each strategy

4 and mode of inheritance (nuclear and/or mitochondrial; ignoring dominance terms as appropriate).

5 Several preliminary simulations were run to check that this parameter space and sample size affords a

6 suitable dataset for subsequent analysis. Most parameters are suited to a range between zero and one

7 (Table 4). Some parameters are better suited to a log-scale (population size, initial frequency and

8 resistance cost) to ensure the random sampling of values that would be considered both qualitatively

9 large and small without being implausible. Lastly, one parameter has no meaningful upper limit

10 (intrinsic birth rate) so a standard log-normal distribution is used, which ensures that the majority of

11 randomly sampled values maintain a stable population size (at the carrying capacity) in the absence of insecticides.

13 The primary method of analysis of simulation data uses conditional inference trees, which is similar to 14 how simulation results are presented using another type of decision tree in Levick et al. (2017). With 15 the comparison of multiple strategies (not just two) for a given measure (e.g. first-to-break), conditional inference trees provide a robust analysis for the categorical classification of when a particular strategy tends to be favoured in different regions of the parameter space. Trees are built and drawn using R:ctree (in the 'partykit' package) (Hothorn et al., 2015), which uses iterative permutation tests in an algorithm that tests the independence between the inputs and output variables and makes a binary split in the variable with the strongest differentiation of output distributions. The iterations that form the tree have a controlled stop when the algorithm can no longer make a split into terminal nodes with $>5 \%$ of the data, which is a control applied for the visualisation of the tree to ensure a manageable number of terminal nodes. Necessarily, there is an element of bias introduced by the choice of any parameter space, but the use of conditional inference trees mitigates against wholescale bias because the meaningful outputs do not rely upon the frequency of a category across the chosen parameter space but rather within statistically different parameter subspaces that are identified algorithmically. Therefore, whilst the frequency of categories must be interpreted with caution (as they depend on the chosen parameter 
space), there is only the potential for bias to enter into the output in determining the precise boundaries

between parameter subspaces where different strategies are more successful. In this way, the use of conditional inference trees is aligned with the aim of understanding how some strategies are favoured over others for a particular measure, which can then provide the basis for insight into why some strategies are favoured over others in different contexts.

The classification process to assemble the output in Levick et al. (2017) first filtered the data to exclude all comparisons where at least one strategy takes longer than the 500 generations of the simulation, and then was either based on a 'hard' cut-off of whichever strategy had the longest time to resistance or biasedly requiring $>20 \%$ longer time to resistance for mixtures (else sequences). Here, to make additional comparisons, non-measured data types that take longer than the 500 generations of the simulation (see Figure 1) are given nominal values that ensure their hierarchical interpretation: where 'Toward Threshold' is set to 1000, 'Away from Threshold' is set to 1500 and 'Extinction' is set to 2000. These simulation-outcome types, or simply "data types", are referred to in detail in the results section. With additional strategies, the classification can include 'sequences' as a lower benchmark but must exclude the 'maximum' upper benchmark because this would mask meaningful comparisons, so comparisons are made between sequences, rotations, mosaics and mixtures. Taking one measure at a time (first-to-break, second-to-break or control-failure), the output variable is assembled by classifying which one or combination of strategies have $>10 \%$ difference (in either direction) for that measure. A combination of strategies is categorised when strategies have $<10 \%$ difference with each other and all have $>10 \%$ difference with all other strategies. This 'soft' cut-off can help avoid misinterpreting the quantitative variation in the measure where strategies have near-equal results. However, this does generate a problem of intransitivity (e.g. where $\mathrm{A}$ is near-equal $\mathrm{B}, \mathrm{B}$ is near-equal $\mathrm{C}$ and $\mathrm{A}$ is not nearequal C), which is best avoided by making fewer comparisons. Consequently, although results can be summarised in a tree that compares all strategies and their combinations at once (as is done in the maintext), special attention is given to a different set of trees where each strategy is examined in isolation and classified as the most successful (>10\% difference than all others), the equally-most successful with one or more other strategy ( $>10 \%$ difference than the worst), the equally-most successful alongside all 
1 other strategies (all $<10 \%$ difference) or not among the most successful (other(s) have $>10 \%$ difference;

2 Supplementary Figures S1-12).

3 It is particularly relevant to the development of new insecticides that there are significant challenges to

4 the design of an ITN with a mixture of insecticides. There are a large number of research and

5 development hurdles to the practical development of an ITN with a mixture, including that insecticides

6 may have different physiochemical properties that are difficult to simultaneously accommodate and/or

7 insecticides may interact antagonistically and so reduce each other's effectiveness. Additionally, there

8 are also criteria to meet, including that the WHO requires that both insecticides are effective on their

9 own, e.g. each with $>80 \%$ mortality in cone assays (where a mosquito is placed in a confined space

10 with the ITN for 3 minutes and mortality is measured 24 hours after this exposure) and also together

11 (World Health Organization, 2001). Lastly, there are also economic hurdles to the success of an ITN with a mixture, both in the funding of research and development (given the other hurdles) and in the manufacturing the end-product. In case mixtures are not possible, the classification of which strategy is favoured is also rerun excluding mixtures (i.e. for sequences, rotations and mosaics) to consider how this alters the results.

Whilst conditional inference trees describe how different strategies are favoured in particular parameter spaces, here there is a special focus in the resistance-management strategy for SC1 and other new insecticides. As such, the secondary method of analysis concerns the results when a new insecticide is used alongside a partner insecticide and in different geographic settings. Due to the uncertainty around the genetic mechanism of resistance for SC1 or any other new insecticide, resistance could involve mutations that affect the target-site or other mutations that bring about changes in metabolism or behaviour, which has different implications for mitochondrial or nuclear inheritance, the extent of resistance restoration (and its dominance) and the resistance cost (and its dominance). Consequently, the only parameter that SC1 or another new insecticide constrains is effectiveness because of the design constraint from WHO criteria, requiring that a new insecticide has $>0.8$ effectiveness to obtain prequalification listing (World Health Organization, 2001, 2020a). A new partner insecticide would be similarly constrained, but the WHO also recommends the use of pyrethroids despite widespread 
1 resistance (World Health Organization, 2012). South and Hastings (2018) estimate the fitness model

2 parameters for pyrethroids using data from an experimental hut study, but this estimation was restricted

3 to $k d r$ (i.e. target-site) resistance, whereas the genetics of resistance to pyrethroids is known to be highly

4 heterogeneous across sub-Saharan Africa (Hancock et al., 2020) and pyrethroids have been shown to

5 have variable effectiveness on different mosquito strains from different locations - even in the same

6 regions of sub-Saharan Africa (Strode et al., 2014; Kleinschmidt et al., 2018). Further, although it is

7 typical for pyrethroids to have lower effectiveness than new insecticides because of pre-existing

8 resistance, this does not imply anything about the genetics of new forms of resistance going forward.

9 Therefore, to assess the restricted parameter space for resistance management with at least one new

10 insecticide, the impact of effectiveness can be assessed by exploring the trend in time to resistance

11 across a focal insecticide's effectiveness and, given a focal insecticide has $>0.8$ effectiveness, across a

12 partner insecticide's effectiveness.

13 Assessing geographic variation also comes with similar problems for assessing the genetics of

14 resistance, but there are qualitative differences between regions on the basis of female exposure. South

15 and Hastings (2018) do not give an estimate of exposure because this cannot easily be quantified by

16 any current data as it is the net result of numerous interacting factors. For example, the major malaria

17 vectors are known to be more zoophilic (animal-feeding) outside of Africa, whilst Africa itself has cooccurring species of which the key transmitters of malaria are more anthropophilic, but it is difficult to quantify zoophily in situ where zoophily is both an evolved factor of ecology and a consequence of host availability in a local area (e.g. see comparisons in Waite et al., 2017). The use of ITNs adds further complication to estimating the effect of zoophily on exposure because ITNs are likely to both select for zoophily within species and cause an ecological shift toward more zoophilic species (Stone \& Gross, 2018). Setting zoophily aside, the most quantifiable aspect of exposure is ITN coverage. Although the distribution of ITNs has aimed for universal coverage to maximize mosquito control (Killeen, 2020), this aim has proved especially difficult to achieve in Africa where malaria burdens are highest. On average across at-risk populations in sub-Saharan Africa in 2018 , whilst $\sim 60 \%$ of households have access to an ITN, only $40 \%$ of households have enough ITNs for all occupants (World Health 
1 Organization, 2019). There is also substantial variation in ITN coverage to at-risk households across

2 sub-Saharan Africa from $>80 \%$ having access to an ITN in their household in Uganda to $<10 \%$ in

3 Gabon. Therefore, although quantitative estimation of female exposure is complicated by numerous

4 underlying factors, to assess the restricted parameter space for resistance management where a focal

5 insecticide has $>0.8$ effectiveness, the qualitative impact of factors like zoophily and coverage leading

6 to high or low female exposure can be assessed by exploring the trend in time to resistance across female 7 exposure. 
Table 4. The parameters and their ranges for the deterministic simulations. Where possible, parameters are randomly sampled across their full range, but some parameters are better suited to a log-scale (population size, initial frequency and resistance cost) and one parameter has no meaningful upper limit (intrinsic birth rate) so a standard log-normal distribution is used (with mean $=0$ and $s d=1$ ). Initial frequency is limited to be within the range $1 / \mathrm{N}$ to $\mathrm{N} / 100$ where $\mathrm{N}$ is population size.

\begin{tabular}{|c|c|c|c|}
\hline \multicolumn{2}{|l|}{ Parameter } & Definition & Range \\
\hline \multicolumn{2}{|c|}{ Population size } & starting population size and carrying capacity & $10^{2}-10^{9}$ \\
\hline \multicolumn{2}{|c|}{ Intrinsic birth rate } & $\%$ population growth rate (in logistic model) & $0-\mathrm{NA}$ \\
\hline \multicolumn{2}{|c|}{ Adult death rate } & $\%$ breeding mosquitoes that die into next generation & $0-1$ \\
\hline \multirow{2}{*}{ Exposure } & Female & $\%$ female mosquitoes that receive a dose & $0-1$ \\
\hline & Male & $\%$ male mosquitoes that receive a dose & $0-1$ \\
\hline \multirow{2}{*}{$\begin{array}{l}\text { Insecticide } \\
\text { effectiveness }\end{array}$} & Insecticide 1 & $\%$ dosed mosquitoes that die from insecticide 1 & $0-1$ \\
\hline & Insecticide 2 & $\%$ dosed mosquitoes that die from insecticide 2 & $0-1$ \\
\hline \multirow{2}{*}{$\begin{array}{l}\text { Initial } \\
\text { frequency }\end{array}$} & Allele A & starting frequency of allele $\mathrm{A}$ & $10^{-9}-10^{-2}$ \\
\hline & Allele B & starting frequency of allele B & $10^{-9}-10^{-2}$ \\
\hline \multirow{2}{*}{$\begin{array}{l}\text { Resistance } \\
\text { restoration }\end{array}$} & Allele A & $\%$ return to baseline fitness with resistance allele $\mathrm{A}$ & $0-1$ \\
\hline & Allele B & $\%$ return to baseline fitness with resistance allele $\mathrm{B}$ & $0-1$ \\
\hline \multirow{2}{*}{$\begin{array}{l}\text { Dominance } \\
\text { of resistance } \\
\text { restoration }\end{array}$} & Allele A & $\%$ resistance restoration in heterozygote with allele $\mathrm{A}$ & $0-1$ \\
\hline & Allele B & $\%$ resistance restoration in heterozygote with allele $\mathrm{B}$ & $0-1$ \\
\hline \multirow{2}{*}{$\begin{array}{l}\text { Resistance } \\
\text { cost }\end{array}$} & Allele A & $\%$ non-dosed mosquitoes that die from carrying allele $\mathrm{A}$ & $10^{-3}-10^{-1 / 2}$ \\
\hline & Allele B & $\%$ non-dosed mosquitoes that die from carrying allele B & $10^{-3}-10^{-1 / 2}$ \\
\hline \multirow{2}{*}{$\begin{array}{l}\text { Dominance } \\
\text { of resistance } \\
\text { cost }\end{array}$} & Allele A & $\%$ resistance cost in heterozygote with allele $\mathrm{A}$ & $0-1$ \\
\hline & Allele B & $\%$ resistance cost in heterozygote with allele $\mathrm{B}$ & $0-1$ \\
\hline
\end{tabular}




\section{$1 \quad$ Results}

2 A parameter space of 17 input parameters was assembled by randomly sampling the parameters for each of the 1 million simulated runs of the model for specific combinations of resistance allele modes of inheritance $(\mathrm{N}=$ nuclear and $\mathrm{M}=$ mitochondrial, giving $\mathrm{NN}, \mathrm{MN}$ and $\mathrm{MM}$ combinations $)$ and strategies (the lower benchmark sequences, the upper benchmark maximum and the three main strategies: rotations, mosaics and mixtures) to record data on the time for the first and second resistance allele to reach $>50 \%$ frequency (first-to-break and second-to-break), the time for the population to recover to $>80 \%$ of its size prior to the application of insecticides (control-failure) and the data types (especially the occurrence of extinction). To avoid any ambiguity, the same random sample of parameters was used for each of the 15 simulations of combinations of inheritance and strategy to make results comparable by inheritance and strategy. The total number of simulations run here is therefore 15 million.

\section{Distributions of the data types}

The results show substantial variability in the simulation-outcome data types across different inheritances, strategies and measures (Figure 1). Based on the change in allele frequency or population size between the start and end of the simulation, the simulated runs can be categorised into:

Successful measurement within 500 generations, as $50 \%$ resistance allele frequency or $80 \%$ of initial population size are reached.

Toward the threshold of change in allele frequency, implying that successful measurement would occur in $>500$ generations).

Away from the threshold of change in allele frequency, implying that allele-frequency change has followed the opposite direction of resistance evolution.

Population extinction, as the female population size drops below one individual leading to extinction of that simulation's population. 
A

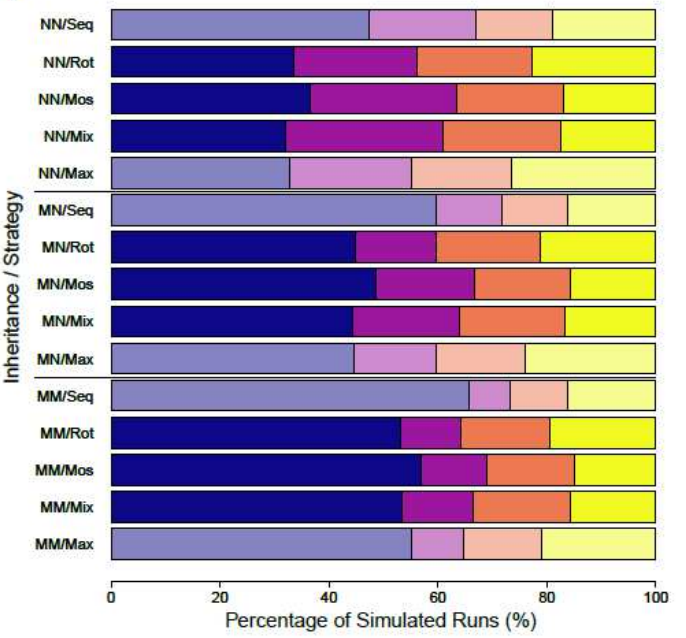

B

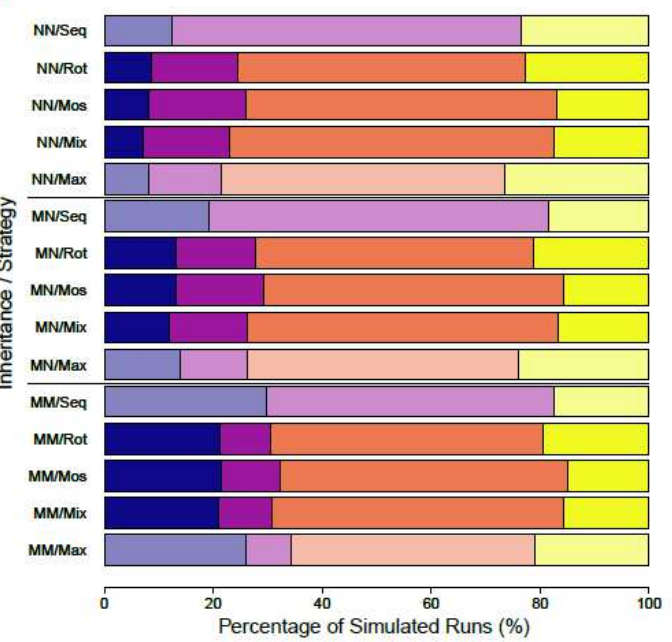

C

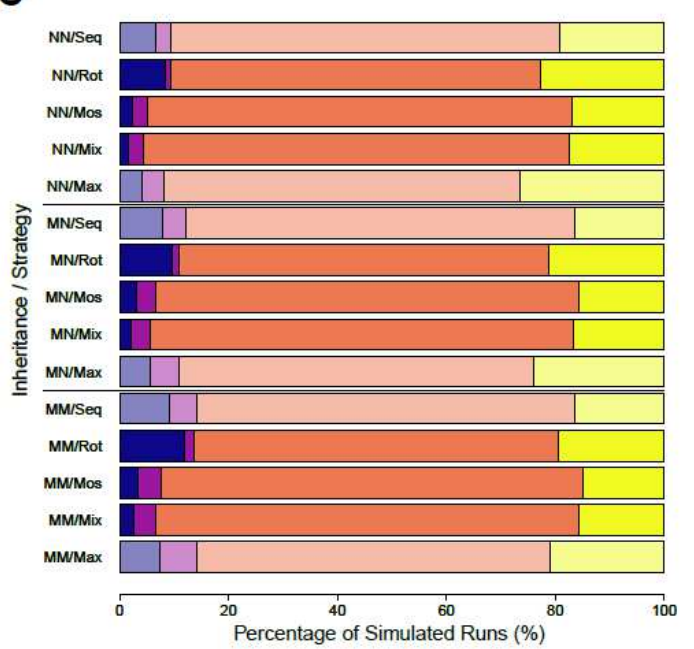

1 million simulated runs: - Successful Measurem Away from Threshol Extinction

NB: Second-to-Break NN/Seq/Away $=83$ MN/Seq/Away $=49$
MM/Seq/Away $=25$

Figure 1. Bar chart of the output of the simulation of 1 million randomly sampled parameter combinations by data type. Simulations are divided between threshold measures (panels A, B and C), mode of inheritance ( $\mathrm{N}=$ nuclear and $\mathrm{M}=$ mitochondrial, giving NN, MN and MM combinations) and strategy (Seq = sequences, lower benchmark; Rot = rotations; Mos = mosaics, Mix = mixtures; Max = maximum, upper benchmark). Simulation runs are classified as 'Successful Measurement' meaning that the threshold statistic was exceeded to give a time to the threshold measurement, 'Toward Threshold' meaning that the resistance allele or population size was approaching the threshold but too slowly to give a measurement, 'Away from Threshold' meaning that resistance allele or population size was decreasing over time (or, for population size, never decreases below $80 \%$ to be able to give a measurement) and 'Extinction' meaning that the female population size drops below 1 (and the simulation terminates). For panel B and the second-to-break measure, the bar chart appears to show no 'Away from Threshold' data types for the sequences strategy but this is due to a low number of simulated runs (with raw numbers given in the NB box), which reflects that the insecticide that is first-to-break has a resistance allele that is placed first in the sequence order. 
1 For all measures (i.e. 'first-to-break', 'second-to-break' and 'control failure'), there is an almost linear

2 trend in data types across inheritances, with nuclear-only inheritance (NN) showing fewer successful

3 measurements and more changes in allele frequency toward the 50\% threshold for measurement,

4 mitochondrial only inheritance (MM) showing a shift toward the opposite pattern and mixed

5 inheritances $(\mathrm{MN})$ showing an intermediate pattern. With mixed modes of inheritance (MN), the

6 insecticide with the resistance allele with mitochondrial inheritance is disproportionately more likely to

7 be the first-to-break (with a mean across strategies of 61.5\%). Across modes of inheritance, the first-to-

8 break measure shows more successful measurements and changes in allele frequency toward the

9 threshold for measurement than the second-to-break measure that shows many more changes in allele

10 frequency away from the threshold for measurement, which reflects the inherent order in the results of

11 these measures (i.e. naturally the second-to-break would take longer than the first-to-break event).

12 Across comparisons, strategies group together with similar distributions of data types. The two strategies with a temporal dimension as insecticide use switches through time (sequences benchmark and rotations) tend to group together, although sequences show an elevated frequency of successful measurements for the first-to-break measure due to applying the strongest selection pressure of any strategy (from one insecticide in constant use) and a highly elevated frequency of changes in allele

17 frequency toward (at the expense of away from) the threshold for measurement for the second-to-break measure because the resistance allele starts from its initial frequency at the point that its insecticide first comes into use (which is a constraint of the use of this benchmark). The other three strategies without the temporal dimension (mosaics, mixtures, maximum) also tend to be grouped together, but the maximum strategy shows an elevated frequency of population extinctions due to applying the strongest initial control of any strategy (from two insecticides at full combined effectiveness).

23 Both the second-to-break and control-failure measures show a large fraction of simulated runs with changes in allele frequency away from the threshold for measurement; this has consequences for strategy comparison, implying that strategies have a more uniform impact on these measures than firstto-break. This is especially problematic for the control-failure measure, which has a large fraction of simulated runs with changes in allele frequency away from the threshold for measurement because the 
1 population size never drops below the threshold of $80 \%$ of initial population size for the recording of a 2 measure of returning to $80 \%$ of population size. Altering the $80 \%$ threshold does not lead to the 3 generation of more data where strategies can be differentiated because such data would simply reveal more space where strategies have a more uniform impact of this measure. The main driver of this result is that the simulation across parameter ranges covers a large fraction of parameter combinations where the initial control that decreases population size is minimal relative to the intrinsic birth rate that increases population size. This is a consequence of the unbiased approach to sampling the parameter space. Furthermore, simply decreasing the average intrinsic birth rate does not provide a solution because this causes a reversion to another problematic outcome in decreasing the frequency of successful measurement of the first- and second-to-break measures through increasing the frequency of extinctions. But, moreover, making a change would imply that the frequency of this data type result is simply an artefact of the model setup, whereas we would argue that it is an informative result in suggesting that strategies have a more uniform impact on control, which is unsurprising given that all the strategies have similar initial control (and, to make strategy comparison fairer, we even ensured that mixtures have the exact same average initial control as rotations and mosaics). Therefore, whilst this data type means that we cannot carry out a very informative analysis on the control-failure measure going forward (although the results of the analysis of this measure are presented in the supplement), the focus of analysis can now shift to where strategies produce a different result, which is in their first-tobreak, second-to-break and probability-of-extinction measures (derived from data types that reflect population control).

Within the data type of 'successful measurement' within 500 generations for the first- and second-tobreak measures (Figure 2), there is a consistent distribution of measured values across inheritance and strategy. Whilst it is rare for a measure to be recorded in a very small number of generations, which reflects the time it takes for a resistance allele to spread from its initial frequency (that can be as high as $1 \%$ ) to $>50 \%$ frequency, it is also rare for a measure to take a large number of generations (nearer 500), which reflects that the distribution is conditional upon successful measurement within 500 generations. The result is a skewed-normal-like distribution, which is more skewed toward zero for the 
1 first-to-break measure than the second-to-break measure because of the inherent order in the results of

2 these measures. Following the same pattern that is observed in the distribution of data types, inheritance

3 produces an almost linear trend across inheritance in both measures, with mitochondrial only

4 inheritance (MM) tending to show a faster time to resistance for both measures, nuclear only inheritance

5 (NN) tending to show a slower time to resistance and mixed inheritances (MN) showing an intermediate

6 pattern between the two extremes. Across strategies, sequences appear to provide a suitable lower

7 benchmark in having a distribution that is obviously lower for both measures in being more skewed

8 toward zero, but the maximum strategy does not appear to obviously set an upper benchmark. This can

9 be explained with reference to data types (Figure 1), where maximum shows an elevated frequency of

10 extinctions, which are excluded from the distribution of the data type for successful measurement

11 (Figure 2). This implies that the distribution of successful measurements shows an underrepresentation

12 of longer times to resistance for the maximum because of extinction. This finding demonstrates that the

13 analysis going forward needs to incorporate multiple data types to accurately describe the results.

14 Across the main strategies, the degree of skew in the distribution toward zero of the first-to-break

15 measure follows the order (more-to-less) of rotations, mosaics and then mixtures, but the distributions

16 are more similar (and variable across inheritance) for the second-to-break measure. 
A

Nuclear Inheritance Only (NN)

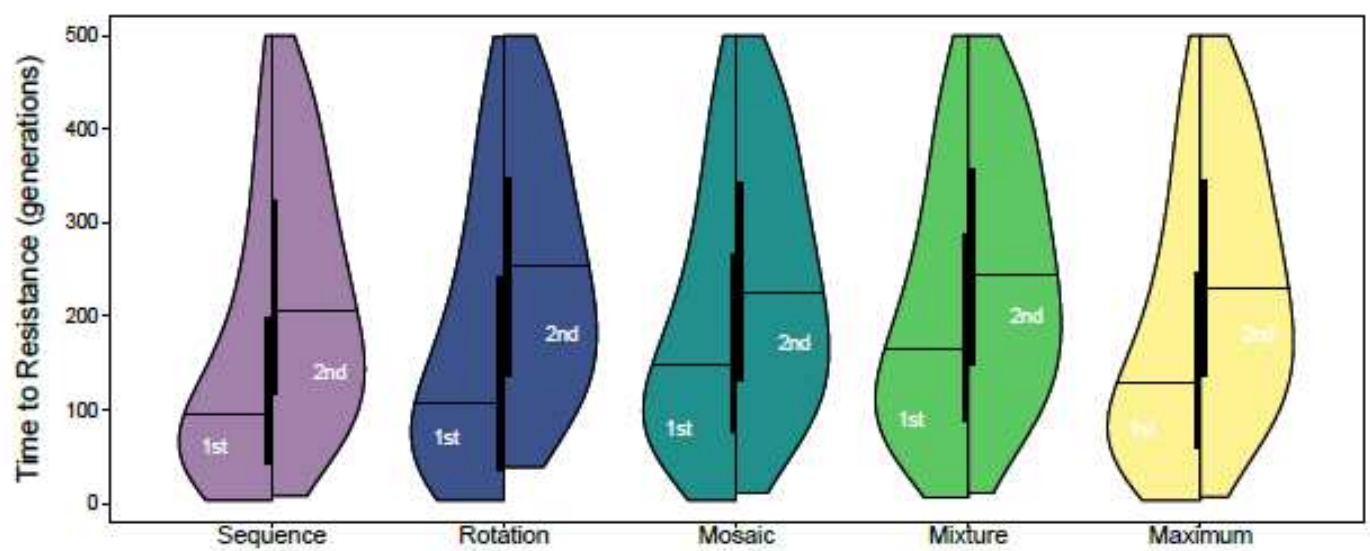

B

Mixed Inheritances (MN)

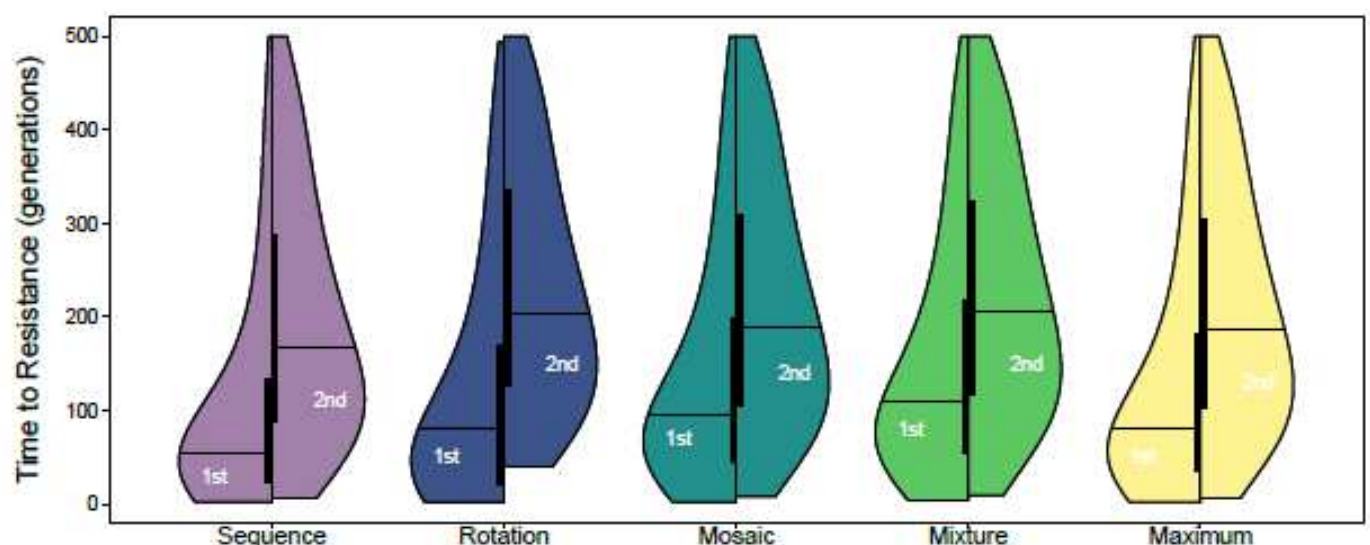

C Mitochondrial Inheritance Only (MM)

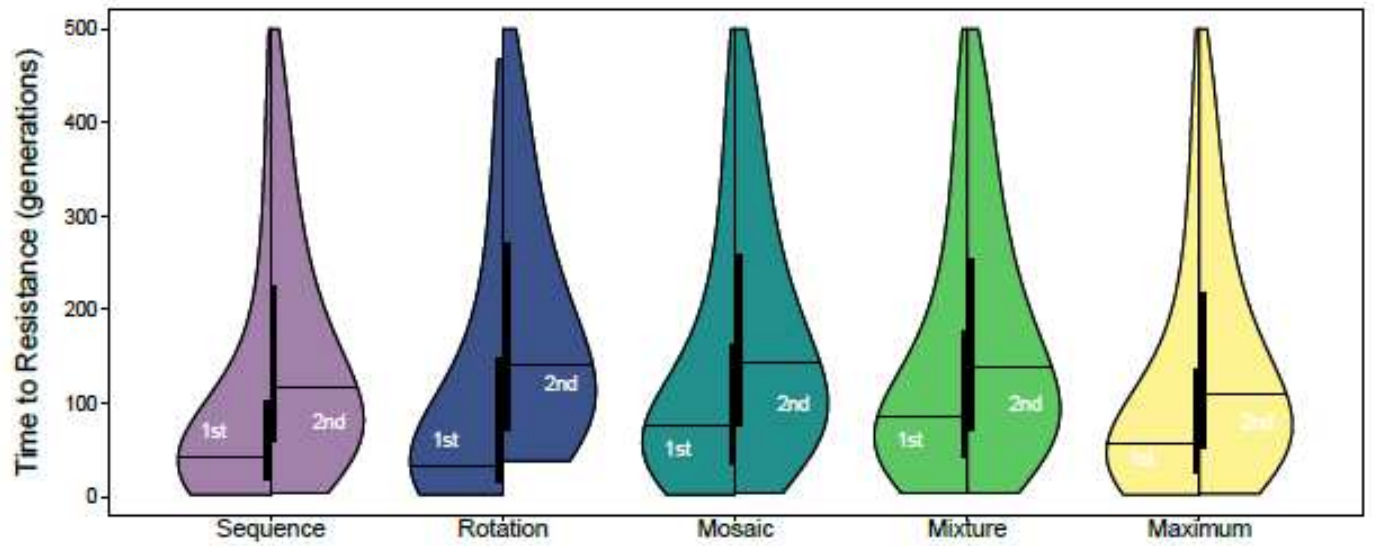

Figure 2. Violin plot of the time to resistance allele exceeding 50\% frequency. The distribution is plotted for the 'Successful Measurement' data type (see Figure 1) with results divided between panels (A, B and C) by the mode of inheritance ( $\mathrm{N}=$ nuclear and $\mathrm{M}=$ mitochondrial, giving $\mathrm{NN}, \mathrm{MN}$ and $\mathrm{MM}$ combinations). Strategies are plotted purple-to-yellow (viridis) by colour with the benchmarks (Sequences and Maximum) given a faded colour. Violins are drawn using R:vioplot with separate lefts for the first-to-break measure (1st) and rights for the second-to-break measure (2nd). Each half-violin has the kernel-density distribution around a box plot, where the vertical bar is drawn between the upper $\left(75^{\text {th }}\right.$ percentile) and lower $\left(25^{\text {th }}\right.$ percentile) quartiles and the horizontal bar is at the median. 


\section{$1 \quad$ Conditional inference trees}

2 Conditional inference trees provide a robust analysis for describing how the quantitative value of a

3 particular parameter influences whether or not a particular strategy tends to be favoured over others.

4 For a given measure, a strategy is favoured when it has $>10 \%$ difference (in either direction) for that

5 measure, which also applies to a combination of strategies when a subset of strategies have $<10 \%$

6 difference with each other and all have $>10 \%$ difference than all other strategies. All data types are

7 included in the analysis using nominal values. For the conditional inference tree comparing the four

8 strategies (sequences, rotations, mosaics and mixtures; excluding maximum as this would mask the

9 other strategies) and their combinations as presented here in the main-text, there is potentially a problem

10 of intransitive comparisons. This can be avoided by making a more explicit comparison of when a

11 particular strategy is favoured outright, in combination with some strategies against others, equally with

12 all other strategies or is not favoured. This approach requires generating one conditional inference tree

13 per inheritance and strategy as is presented in the supplement (Supplementary Figures S1-12). Yet, the

14 categorisation process reveals that only five classifications are needed to describe the data: ' $=$ ' where

15 all strategies have $<10 \%$ difference, ' $\mathrm{CX}$ ' where mosaics $(\mathrm{C})$ and mixtures $(\mathrm{X})$ have $<10 \%$ difference

16 but $>10 \%$ difference than rotations (R) or sequences, 'CXR' where mosaics (C), mixtures (X) and

17 rotations (R) have $<10 \%$ difference but $>10 \%$ difference than sequences, ' $R$ ' where rotations have

$18>10 \%$ difference than all other strategies and ' $\mathrm{X}$ ' where mixtures have $>10 \%$ difference than all other

19 strategies. Consequently, with just five comparisons, the results for the summary tree of all comparisons

20 presented here are a good approximation of the cumulative results of the trees-by-strategy for a given

21 inheritance (Figure 3). Additionally, the summary of results presented here is only for the first-to-break

22 measure, but the second-to-break and control-failure measures are detailed in summary and full figures

23 in the supplement (Supplementary Figures S13-42). 

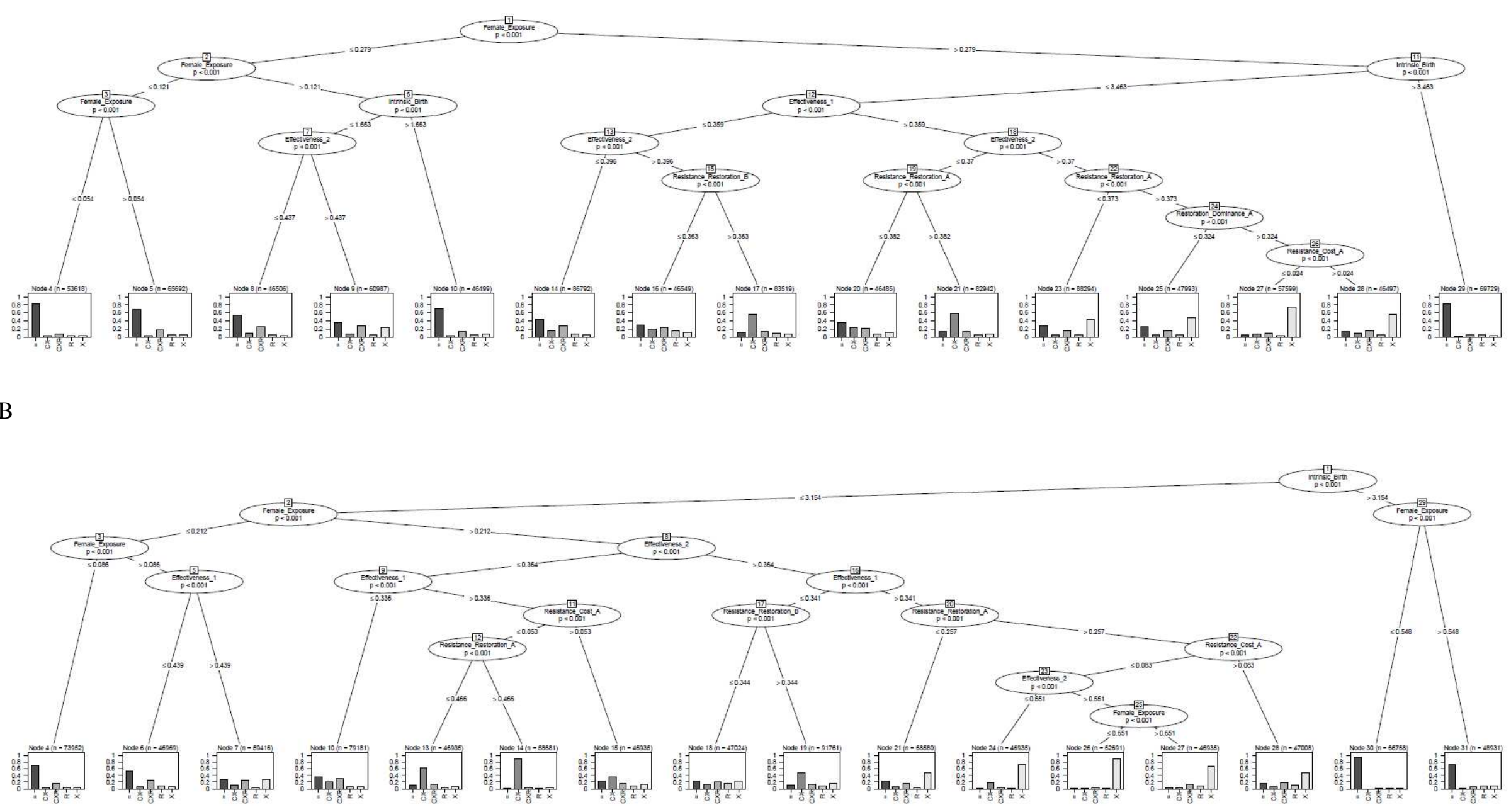


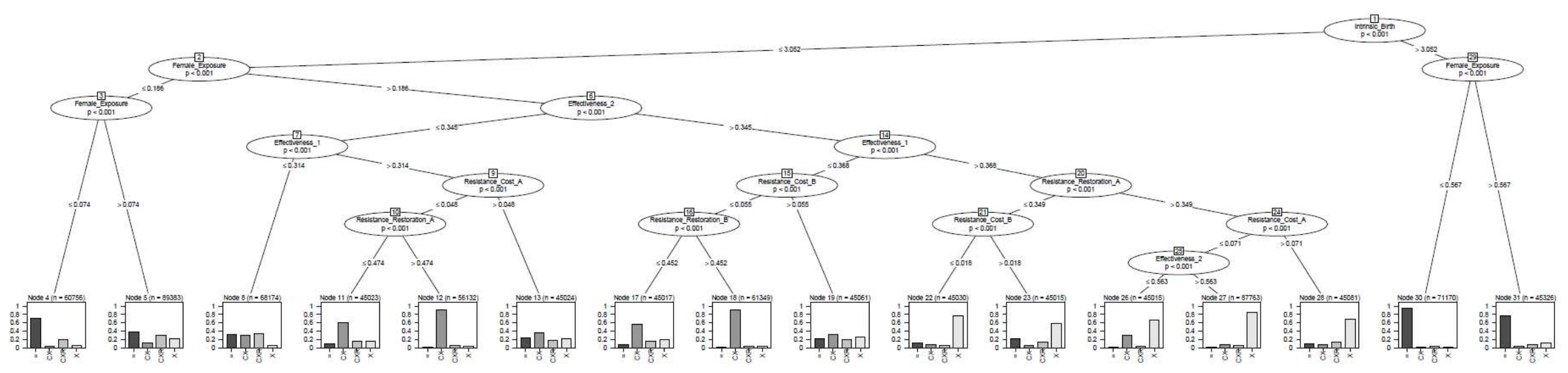

3 Figure 3. Conditional inference trees for the first-to-break measure by each combinatorial mode of inheritance $(\mathrm{N}=$ nuclear and $\mathrm{M}=$ mitochondrial, giving combinations for 4 panel A as NN, panel B as MN and panel C as MM). The simulation data on the first-to-break for each strategy is classified into a categorical variable to describe whether one 5 or multiple strategies have $>10 \%$ difference (in either direction) in their first-to-break measure, including the 'sequences' lower benchmark but excluding the 'maximum' upper 6 benchmark (because this would mask meaningful comparisons). Non-measured data types (see Figure 1) are given nominal values that ensure their hierarchical interpretation: 7 where 'Toward Threshold' is set to 1000, 'Away from Threshold' is set to 1500 and 'Extinction' is set to 2000. Data classifications are given for all strategies and their 8 combinations, but only five classifications are needed to describe the data: ' $=$ ' where all strategies have $<10 \%$ difference, ' $\mathrm{CX}$ ' where mosaics (C) and mixtures (X) have $<10 \%$ 9 difference but $>10 \%$ difference than rotations (R) or sequences, 'CXR' where mosaics (C), mixtures (X) and rotations (R) have $<10 \%$ difference but $>10 \%$ difference than 10 sequences, ' $\mathrm{R}$ ' where rotations have $>10 \%$ difference than all other strategies and ' $\mathrm{X}$ ' where mixtures have $>10 \%$ difference than all other strategies. The conditional inference 11 tree is used to partition the data classification output based on the parameter space inputs based on the 1 million randomly sampled parameter combinations for the 17 parameters

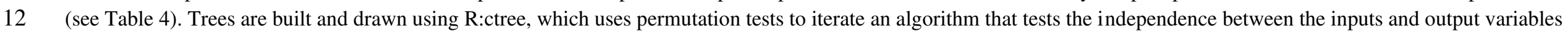

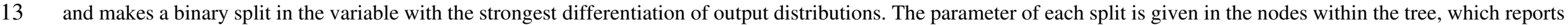
the parameter (as per Table 4) and the $p$-value of the independence test; the quantitative place of the split in the parameter itself is recorded in the line between nodes. The iterations that form the tree stop when algorithm can no longer make a split into terminal nodes with $>5 \%$ of the data, which is a control applied for the visualisation of the tree to ensure a manageable number of terminal nodes. The distributions of data classification are given in the terminal nodes as a bar chart, where the $y$-axis describes the proportion of data points. 
1 Across the conditional inference trees for the different modes of inheritance, the results are remarkably

2 consistent for the first-to-break measure. All strategies tend to be near-equally favoured (=) when

3 female exposure is $<0.3$, which is logical because this would most likely represent a scenario where the

4 balance of the conditional advantage to a female from resistance restoration under exposure to an

5 insecticide is offset by the unconditional disadvantage of the resistance cost. Indeed, this explanation

6 can be confirmed as a large percentage of simulated runs (means: $\mathrm{NN}=70.8 \%, \mathrm{MN}=61.2 \%, \mathrm{MM}=$

$753.4 \%$ ) show that the change in allele frequency is either toward or away from the threshold when

8 female exposure is $<0.3$. Additionally, all strategies tend to be near-equally favoured (=) when female

9 exposure is $>0.3$ and the intrinsic birth rate is $>3$, which corresponds to an extremely high birth rate

10 with unstable population dynamics such that a large percentage of simulated runs end in extinction

11 (means: $\mathrm{NN}=70.5 \%, \mathrm{MN}=71.2 \%, \mathrm{MM}=73.9 \%$ ). Mixtures $(\mathrm{X})$ tend to be favoured outright when

12 female exposure is $>0.3$, the intrinsic birth rate is $<3$ and the effectiveness of both insecticides is $>0.35$.

13 When one or other insecticide has effectiveness $>0.35$, then mixtures tend to be favoured alongside mosaics $(\mathrm{CX})$ or mosaics and rotations $(\mathrm{CXR}$, which is all the strategies apart from the sequences benchmark). In this parameter space, mixtures and mosaics tend to be favoured (CX) when the insecticide with higher effectiveness ( $>0.35)$ also has higher resistance restoration $(>0.4)$. The secondto-break measure also shows a related pattern across inheritances, all strategies tend to be near-equally favoured (=) when the intrinsic birth rate is $>3$, where the extremely high birth rate leads to unstable population dynamics that often end in extinction (means: $\mathrm{NN}=78.2 \%, \mathrm{MN}=79.2 \%, \mathrm{MM}=80.0 \%$ ). Alternatively, when the intrinsic birth rate is $<3$, mosaics, mixtures and rotations (CXR) are favoured over sequences. In contrast to both the time to resistance measures, the control-failure measure shows no detectable pattern across the parameter space, which is simply because all strategies are near-equally favoured $(=)$.

In case mixtures are not practically possible, the classification can be rerun excluding mixtures (i.e. for sequences, rotations and mosaics) to consider how this alters the conditional inference tree results (see Supplementary Figures S43-78). In short, where mixtures would have been favoured outright (X), instead mosaics tend to be favoured outright (C). For the first-to-break measure, all strategies tend to 
1 be near-equally favoured $(=)$ when the female exposure $<0.3$ or female exposure is $>0.3$ and the intrinsic

2 birth rate is $>3.5$. When female exposure is $<0.3$ and the intrinsic birth rate is $<3.5$, mosaics tend to be

3 favoured outright (C) when the effectiveness of at least one insecticide is $>0.45$, else mosaics and

4 rotations are jointly favoured (CR).

$6 \quad$ Scenarios of new insecticides

7 To provide a quantitative estimation of the difference between strategies across the relevant parameter 8 space for the possibilities for the deployment of new insecticides, the broad trends in the parameter space for all of the different strategies including both benchmarks can be examined across key parameters. The choice of a new insecticide and its partner restricts the parameter space along the axes of the effectiveness of both insecticides, where a new insecticide (including SC1) has high effectiveness $>0.8$ from WHO guidelines (World Health Organization, 2001) whereas pyrethroids can have variable effectiveness within and between localities (Strode et al., 2014; Kleinschmidt et al., 2018; Hancock et al., 2020). Different geographic regions may have different genetic mechanisms of resistance, but this is unpredictable and so geographic variation is only interpreted as restricting the parameter space along the axes of female exposure that is primarily understood as reflecting mosquito zoophily and/or ITN coverage. Consistent with conditional inference trees, there is little distinction between strategies for the second-to-break and control-failure measures (Supplementary Figures S13-42), and so results presented here in the main-text focus on the probability of resistance and time to the first-to-break. The probability of resistance describes the fraction of results of the data types for the first-to-break measure that are either 'Successful Measurement' or 'Toward Threshold' where allele frequencies are changing in the direction of resistance.

Across modes of inheritance, nuclear only $(\mathrm{NN})$ and mitochondrial only $(\mathrm{MM})$ inheritance show a similar variability in the probability of resistance and time to first-to-break across the parameters of effectiveness and female exposure, but with mitochondrial only inheritance (MM) showing a greater probability of resistance and shorter time to first-to-break. Like for the conditional inference trees, mixed inheritances $(\mathrm{MN})$ shows an intermediate pattern across effectiveness parameters, which is closer 
1 to nuclear only inheritance $(\mathrm{NN})$ with lower values of effectiveness and closer to mitochondrial only

2 inheritance $(\mathrm{MN})$ with higher values of effectiveness. This is a consequence of the insecticide with the

3 resistance allele with mitochondrial inheritance being more likely to be the first-to-break when the

4 corresponding resistance allele has higher effectiveness. In contrast, mixed inheritances (MN) has a

5 pattern across female exposure that is much closer to mitochondrial only inheritance (MM), which

6 reflects the high probability (with a mean across strategies of $69.0 \%$ ) that the insecticide with the

7 mitochondrial resistance allele is the first-to-break.

8 Across strategies, the same patterns that were observed in the conditional inference trees can be given

9 a rough quantitative estimation (Figure 4, 5, 6). Again, there is a division in the shape of results among

10 strategies based on the temporal dimension of insecticide switching through time, with sequences and

11 rotations showing a similar variability in the probability of resistance and time to first-to-break across

12 the parameters of effectiveness and female exposure, and mosaics, mixtures and maximum showing a

13 similar variability (albeit that mosaics show the opposite trend across partner insecticide effectiveness

14 for the time to first-to-break). Across all strategies and the probability of resistance and time to first-to-

15 break (panels A, D), there is a similar pattern of results across focal insecticide effectiveness with a

16 higher probability of resistance at intermediate effectiveness, which reflects the balance of data types

17 (Figure 1) at the extremes of 'Away from Threshold' with lower effectiveness $<0.3$ and 'Extinction'

18 with higher effectiveness $>0.5$. In comparison between strategies, mosaics and mixtures tend to perform

19 similarly with lower effectiveness $<0.3$, but with mosaics having a higher probability of resistance and

20 shorter time to first-to-break with higher focal insecticide effectiveness. Rotations have a lower

21 probability of resistance and a shorter time to first-to-break than mosaics and mixtures. The quantitative

22 differences between strategies do not matter for directly addressing the question using a strategy to

23 delay the evolution of resistance because WHO guidelines (World Health Organization, 2001) require

24 that an insecticide has $>0.8$ effectiveness, but this does indicate what this guideline entails. 
A

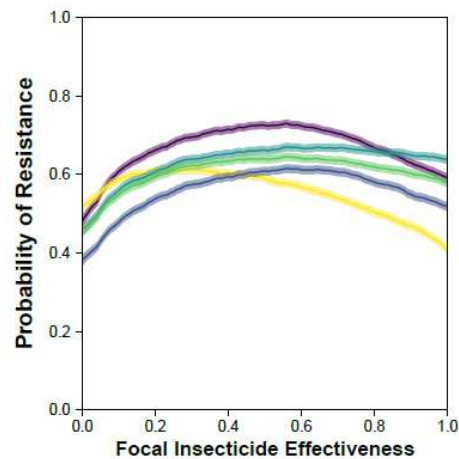

D

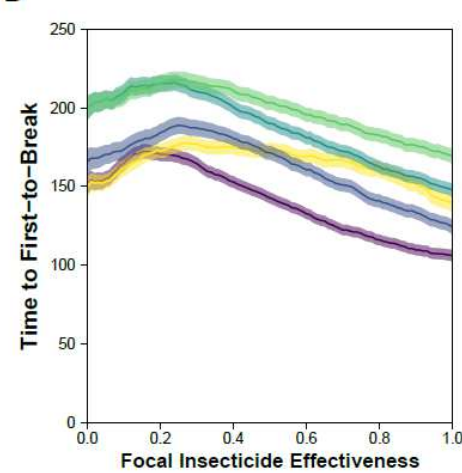

B

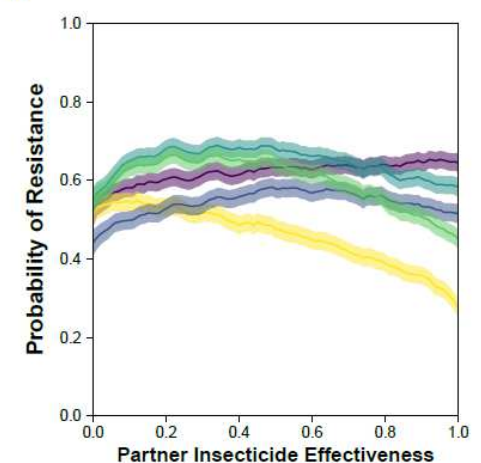

E

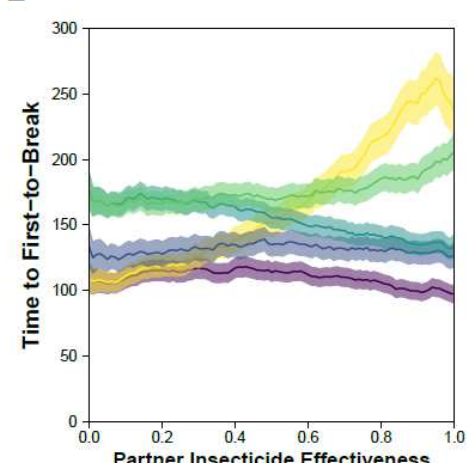

C

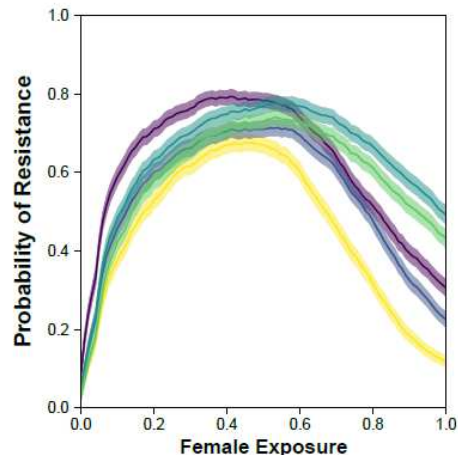

F

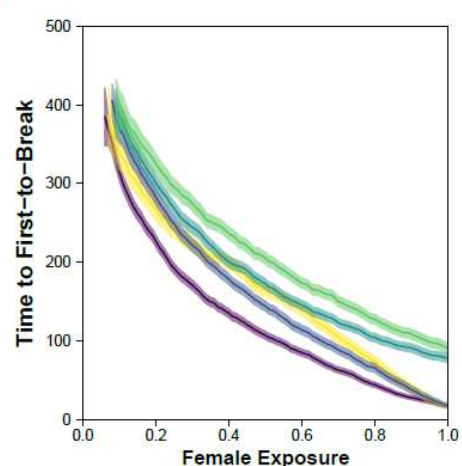

- Sequences

- Rotations

- Mosaics

- Mixtures

Figure 4. Relationship between insecticide choice and geographic location on probability of resistance and time to first-to-break with nuclear only inheritance (NN). Insecticides differ by their effectiveness and geographies differ by their female exposure. The probability of resistance describes the fraction of all simulated runs where the data type is 'Successful Measurement' or 'Toward Threshold' (see Figure 1). The time to first-to-break is calculated from the 'Successful Measurement' data type only. In each panel, the bold-colour lines (per strategy purple-to-yellow; R:viridis) come from partitioning the y-axis parameter from the simulations by the x-axis parameter into 101 rounded bins and calculating the mean of the $\mathrm{y}$-axis measure per bin; a $k=5$ backward-tail moving average is used to smooth the mean-line. Around each bold-colour line, there is a transparentshading of the same colour that describes the $95 \%$ confidence intervals for the mean $( \pm 1.96 * S E)$, which is also smoothed with a $k=5$ backward-tail moving average. In panels $\mathrm{A}$ and $\mathrm{D}$, the $\mathrm{X}$-axis is arbitrarily designated for a focal insecticide as effectiveness 1 , as if it were a new insecticide. In panels B and E, effectiveness 1 is assumed to be $>0.8$ (in accordance with WHO guidelines for new ITNs), and the $\mathrm{x}$-axis is then for a partner insecticide. In panels $\mathrm{C}$ and $\mathrm{F}$, effectiveness 1 is also assumed to be $>0.8$, and the $\mathrm{x}$-axis is then for fem ale exposure. 
A

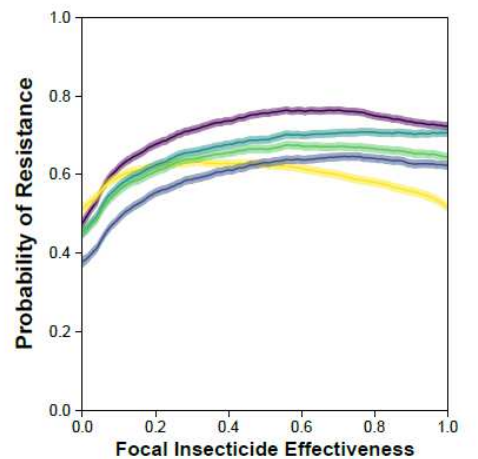

D

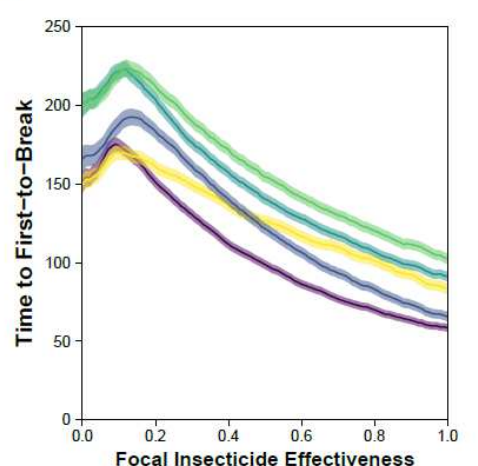

B

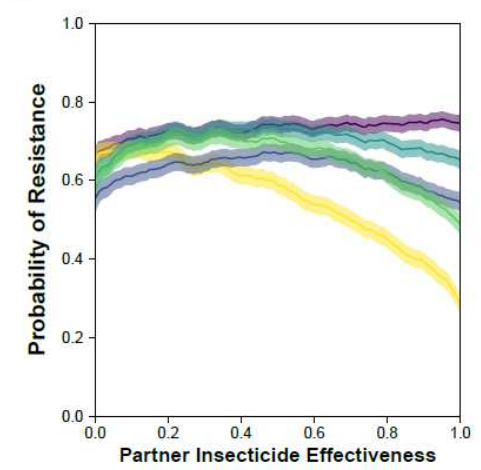

E

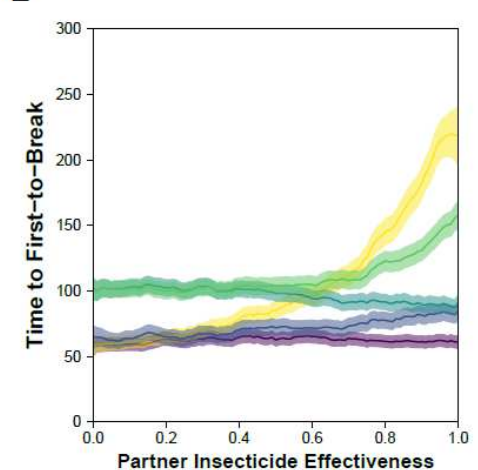

c

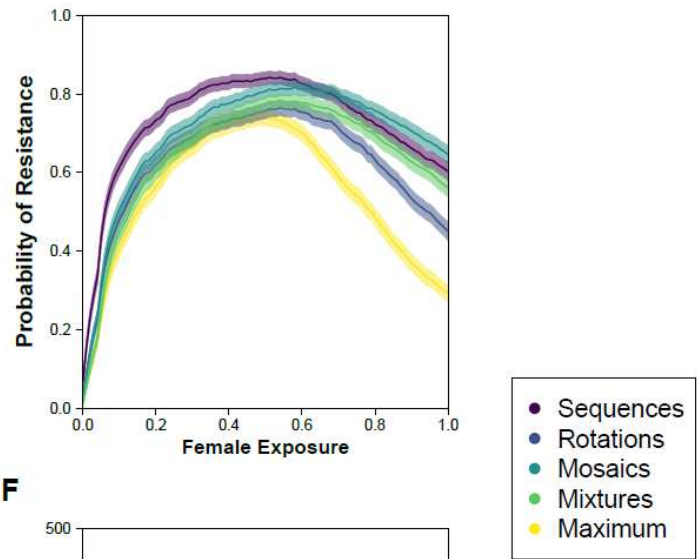

Rotation

Mixtures

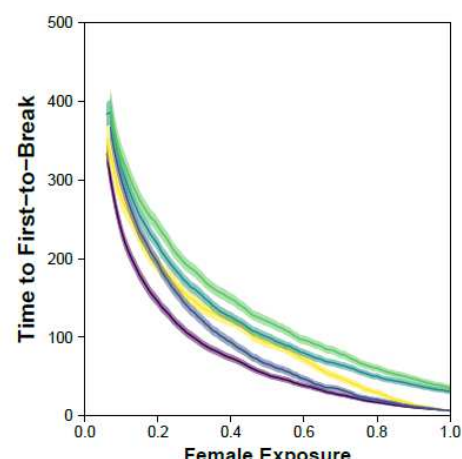

Figure 5. Relationship between insecticide choice and geographic location on probability of resistance and time to first-to-break with mixed inheritance (MN). Insecticides differ by their effectiveness and geographies differ by their female exposure. The probability of resistance describes the fraction of all simulated runs where the data type is 'Successful Measurement' or 'Toward Threshold' (see Figure 1). The time to first-to-break is calculated from the 'Successful Measurement' data type only. In each panel, the bold-colour lines (per strategy purple-to-yellow; R:viridis) come from partitioning the y-axis parameter from the simulations by the $\mathrm{x}$-axis parameter into 101 rounded bins and calculating the mean of the $y$-axis measure per bin; a $k=5$ backward-tail moving average is used to smooth the mean-line. Around each bold-colour line, there is a transparentshading of the same colour that describes the $95 \%$ confidence intervals for the mean $( \pm 1.96 * S E)$, which is also smoothed with a $k=5$ backward-tail moving average. In panels $\mathrm{A}$ and $\mathrm{D}$, the $\mathrm{x}$-axis is designated for the insecticide that corresponds to the mitochondrial inheritance of resistance as effectiveness 1 , as if it were a new insecticide. In panels $\mathrm{B}$ and $\mathrm{E}$, effectiveness 1 is assumed to be $>0.8$ (in accordance with WHO guidelines for new ITNs), and the $\mathrm{x}$-axis is then for a partner insecticide. In panels $\mathrm{C}$ and $\mathrm{F}$, effectiveness 1 is also assumed to be $>0.8$, and the $\mathrm{x}$-axis is then for female exposure. 
A

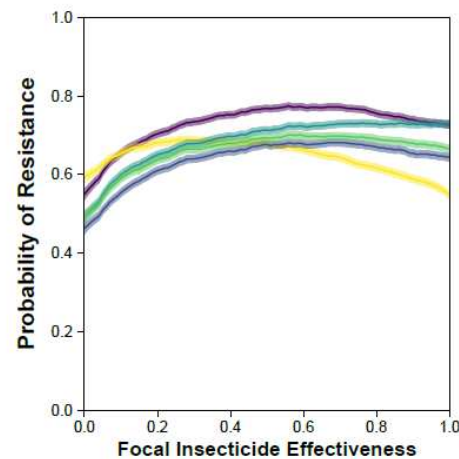

D

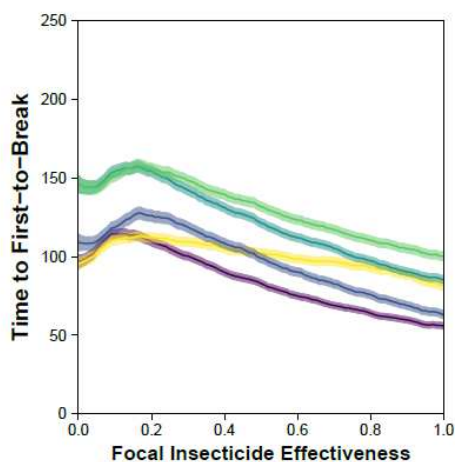

B

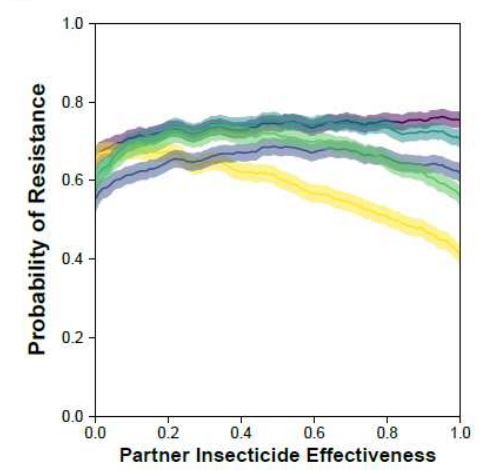

E

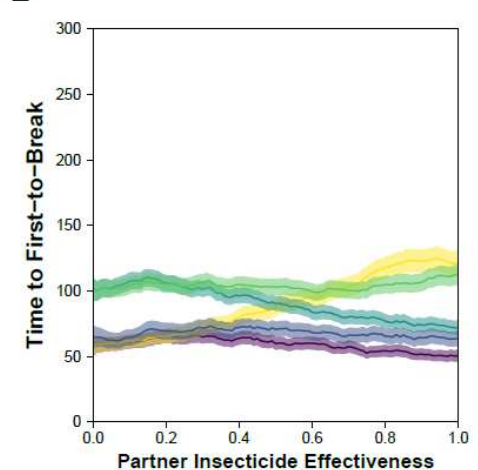

c

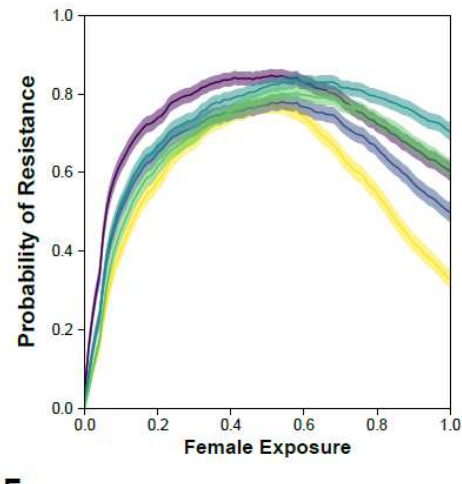

F

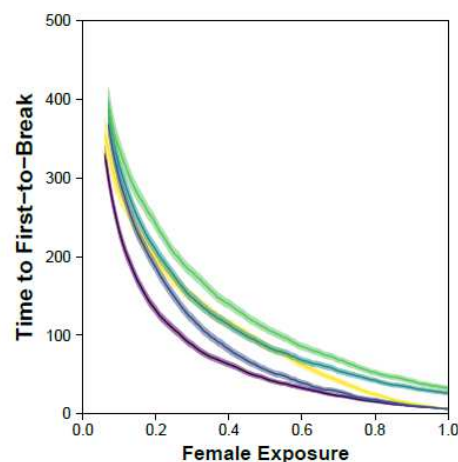

- Sequences

- Mosaics

- Mosaics

Mixtures

Figure 6. Relationship between insecticide choice and geographic location on probability of resistance and time to first-to-break with mitochondrial only inheritance (MM). Insecticides differ by their effectiveness and geographies differ by their female exposure. The probability of resistance describes the fraction of all simulated runs where the data type is 'Successful Measurement' or 'Toward Threshold' (see Figure 1). The time to first-to-break is calculated from the 'Successful Measurement' data type only. In each panel, the bold-colour lines (per strategy purple-to-yellow; R:viridis) come from partitioning the y-axis parameter from the simulations by the $\mathrm{x}$-axis parameter into 101 rounded bins and calculating the mean of the y-axis measure per bin; a $k=5$ backward-tail moving average is used to smooth the mean-line. Around each bold-colour line, there is a transparent-shading of the same colour that describes the $95 \%$ confidence intervals for the mean $( \pm 1.96 * S E)$, which is also smoothed with a $k=5$ backward-tail moving average. In panels A and D, the X-axis is arbitrarily designated for a focal insecticide as effectiveness 1 , as if it were a new insecticide. In panels B and E, effectiveness 1 is assumed to be $>0.8$ (in accordance with WHO guidelines for new ITNs), and the X-axis is then for a partner insecticide. In panels C and F, effectiveness 1 is also assumed to 10 be $>0.8$, and the $\mathrm{x}$-axis is then for female exposure. 
1 There is less variability across the trend line of partner insecticide effectiveness and more variability in

2 estimating the trend line (panels B, E), which is partly because the parameter space is restricted to assume a new focal insecticide with effectiveness $>0.8$. Sequences and rotations are especially uniform across partner insecticide effectiveness, with rotations increasing the time to first-to-break compared to the sequences benchmark (that is like a non-strategy negative control) by $\sim 25 \%$, which is roughly equivalent to an additional two years of insecticide susceptibility from a baseline of eight years. Again, mosaics and mixtures tend to perform similarly with low effectiveness $<0.3$, which increases the time to first-to-break compared to the sequences benchmark by $\sim 50 \%$ or an additional four years of insecticide susceptibility. With higher partner insecticide effectiveness, mosaics show a decrease in the time to first-to-break to become very similar to rotations, whereas mixtures show an increase in the time to first-to-break of up to $\sim 100 \%$ or an additional eight years of insecticide susceptibility. Interestingly, the maximum benchmark, which is how mixtures are attributed in Levick et al. (2017), performs similarly to sequences with low partner effectiveness $<0.3$, which is because the time to first-to-break solely reflects the focal insecticide effectiveness; but, increases with partner insecticide effectiveness to delay resistance by up to $150 \%$ or an additional twelve years on the sequences benchmark.

Whilst there is more variability across female exposure (panels C, F), strategies are more uniform. Sequences both show a higher probability of resistance and lower time to first-to-break than other strategies with lower female exposures, but all other strategies are similar. At higher female exposures, the main strategies have quantitatively different probabilities of resistance that follow a predictable order with mosaics having the highest probability of resistance, mixtures next highest and then rotations; but the difference between strategies narrows with more resistance alleles with mitochondrial inheritance. At higher female exposures for time to first-to-break, rotations increasingly perform similarly to sequences whilst mixtures increasingly perform similarly to mosaics (at a longer time to first-to-break). It is difficult to determine the quantitative consequences of higher female exposure because of competing effects on the probability of resistance and time to first-to-break. At practically plausible levels of female exposure $(<0.8)$, the probabilities of resistance between strategies are not so dissimilar (e.g. at female exposure of $\sim 0.4$ ), where mixtures show an increase in the time to first-to- 
1 break on sequences of $\sim 100 \%$, mosaics up to $\sim 75 \%$ and rotations of $\sim 25 \%$, which is equivalent to seven,

2 five and one additional years of insecticide susceptibility respectively. With higher female exposure at $3 \sim 0.6$, rotations are very similar to sequences, but mixtures and mosaics are more dissimilar with a $4 \sim 150 \%$ and $\sim 100 \%$ increase in the time to first-to-break that only amounts to an additional five and

5 three years of insecticide susceptibility respectively. Higher female exposure is expected in settings

6 with highly anthropophilic mosquito species and contexts such as sub-Saharan Africa (Stone \& Gross,

7 2018) and/or high ITN coverage such as in more affluent African nations (World Health Organization,

8 2019). With lower female exposure at $\sim 0.1$, the main strategies are hard to distinguish but all show a

$9<10 \%$ increase in the time to first-to-break on sequences that represents up to an additional three years

10 of insecticide susceptibility. Lower female exposure is expected in settings with highly zoophilic

11 mosquito species and contexts such as India (Stone \& Gross, 2018) and/or low ITN coverage such as

12 in regions with more isolated rural communities (Killeen, 2020).

14 Discussion

15 Against the backdrop of widespread resistance to existing technologies, the programme to eradicate 16 malaria is at a critical juncture as new insecticides enter their final stages of development for use via 17 the main tool in the fight against malaria in insecticide-treated bed-nets (ITNs). The near-synchronous development of these new insecticides offers the current opportunity to build resistance management into mosquito control methods and maximize the chance of eradicating malaria. Here, building directly on existing modelling (Levick et al., 2017; South \& Hastings, 2018; South et al., 2019), resistancemanagement strategies using multiple insecticides are compared to suggest how to deploy combinations of available and new insecticides on bed-nets for maximum impact. Although the strategy comparison considers what to do with any new insecticide, special attention to SC1, which is the only new insecticide that is known to the authors in having a strobilurin-like chemistry that is unusual for having a target-site that is encoded in the mitochondrial genome. Massive simulations across randomly sampled sets of parameters are run to compare basic strategy concepts for two insecticides (of rotations, mosaics and mixtures under the assumption of equal initial control) against benchmarks (a 'non-strategy' 
1 minimum of a sequence of one insecticide at a time and a 'best-case' maximum where an exposed

2 mosquito gets the full impact of both insecticides simultaneously). Given that strategies are setup for

3 control equivalence to make the comparison 'fairer', (unsurprisingly) the results are only appreciably

4 different for the measures of the time until the first and second resistance allele reach $>50 \%$ frequency

5 (or break).

6 For the time until the second-to-break, each strategy nearly always has $>10 \%$ difference than a non-

7 strategy (i.e. sequences) but $<10 \%$ difference amongst each other, which favours the use of any strategy.

8 Yet, strategies tend to lead to much more variability in the time until the first-to-break. The non-strategy

9 of using insecticides in sequence has $<10 \%$ difference to any strategy (rotations, mosaics or mixtures)

10 for the time to the first-to-break when the exposure of female mosquitoes to ITNs is low $(<30 \%)$ across

11 combinations of resistance allele inheritance (Figure 3). Low female exposure would arise in contexts with more zoophilic mosquitoes, such as in rural India (Stone \& Gross, 2018), and/or lower ITN coverage, such as in inaccessible regions of sub-Saharan Africa (World Health Organization, 2019). However, malaria eradication programmes should aim for universal coverage to provide communitywide protection from vector control (World Health Organization, 2012; Killeen, 2020), so most deployment scenarios can assume higher female exposure (>30\%). In this context, a strategy (rotations, mosaics or mixtures) leads to at least $>10 \%$ difference in the time until the first-to-break. Mixtures tend to have $>10 \%$ difference than the other strategies when the effectiveness of both insecticides is higher (>35\%; Figure 3). As a new insecticide is required by WHO guidelines to have an effectiveness that is $>80 \%$ in a standard cone assay (World Health Organization, 2001), using SC1 or another new insecticide alongside a partner in a mixture increases the time to first-to-break by between $50 \%$ and $100 \%$ (or +4 and +8 years from the estimated and relative baseline of non-strategy taking 8 years) depending on whether the partner insecticide has lower or higher effectiveness (Figures 4, 5, 6 panel E). When one or other insecticide has higher effectiveness ( $>35 \%$ ), then mixtures tend to have $<10 \%$ difference than mosaics but $>10 \%$ difference than rotations or a non-strategy (Figure 3). Consequently, using SC1 or another new insecticide alongside a partner in a mosaic increases the time to first-to-break by between $50 \%$ and $25 \%$ (or +4 and +2 years) depending on whether the partner insecticide has lower 
1 or higher effectiveness (Figures 4, 5, 6 panel E). By contrast, a rotation uniformly increases the time to

2 first-to-break by $25 \%$ (or +2 years) irrespective of the effectiveness of the partner insecticide. Given

3 that one insecticide has high effectiveness $(>80 \%)$, there is some variability in the quantitative

4 difference between strategies across female exposure (Figures 4, 5, 6 panel F), which could imply that

5 different strategies might be suitable in geographic contexts where different levels of ITN coverage are

6 obtainable. However, differences between strategies still supply a strong signal with high focal

7 insecticide effectiveness $(>80 \%)$ to afford the same pattern of results across partner insecticide

8 effectiveness, which become more pronounced with higher female exposures. Therefore, although

9 different strategies are favoured under different parameter combinations (Figure 3), the minimal

10 restriction of WHO criteria on insecticide effectiveness is enough to suggest that mixtures tend to

11 produce the greatest delay in the evolution of resistance across relevant parameter combinations.

12 Using the same parameter framework, the modelling presented here supports a similar result as in Levick et al. (2017) but with some important differences. First, the simulations that are run here are more massive ( 1 million $v s$ 10,000), which introduces less variability into the outputs. Second, the model is run in a way that explicitly categorises data types, which extracts additional information from a run where resistance does not arise within the 500 generations of the simulation. Third, more strategies are compared with greater concern for making a 'fair' comparison, using the additional consideration of population size to ensure that strategies have the same average initial control, which helps to more distinctly isolate the effects of differences between strategy concepts. Consequently, when Levick et al. (2017) report that mixtures tend to be favoured over sequences (with $>20 \%$ difference) in the time to first-to-break when female exposure is $<60 \%$ (and, by the range of parameter, $>10 \%$ ) and effectiveness of both insecticides is $>70 \%$, our simulations and their analysis affords us confidence in clarifying this result. Mixtures are not implemented in the same way as in Levick et al. (2017) in order to make all strategies that are compared here have equal initial control; although the pattern of results are similar, the quantitative differences between mixtures here and in Levick et al. (2017), which is implemented in the maximum benchmark, are substantial (Figure 4). The results presented here do not support the suggestion that mixtures tend to be favoured over sequences when female exposure is $<60 \%$. As the 
1 analysis in Levick et al. (2017) excludes comparisons where resistance does not arise within 500

2 generations for either mixtures or sequences, this biases results against mixtures for higher female 3 exposures because a mixture is more likely to delay the time until resistance to be $>500$ generations

4 (compare sequences and maximum in Figure 1, 4 panel C). Consequently, the restriction of mixtures

5 being favoured to lower female exposure $<60 \%$ is likely to be an artefact of the strategy comparison in

6 Levick et al. (2017). The results presented here do support the general finding that higher insecticide

7 effectiveness tends to favour mixtures over other strategies, but the threshold is lower at $>35 \%$

8 effectiveness. Moreover, mixtures and mosaics tend to be favoured over sequences when one or other

9 insecticide has effectiveness $>35 \%$, which is a marked relaxation of this limit. As such, when mixtures

10 are excluded from strategy comparison (e.g. were they not to be possible for manufacturing reasons),

11 mosaics tend to be favoured in their stead. Therefore, whilst our results support the general findings

12 about high effectiveness in Levick et al. (2017) that is focused on in subsequent analysis (South \&

13 Hastings, 2018; South et al., 2019), especially in contrast to the forerunning ideas from interpretation

14 of the findings in Curtis (1985), the extensions that are implemented within the same parameter

15 framework for two insecticides enable us to clarify that temporally-invariable strategies

16 (mixtures/mosaics) tend to be favoured over temporally-variable strategies (rotations/sequences) in this

17 model. In strategic terms, this suggests that the advantages of simultaneous selective pressures tend to

18 outweigh the advantages of variable selective pressures.

19 What strategy does the modelling suggest should be adopted for SC1 and other new insecticides?

20 Although different strategies can be favoured in different contexts (Figure 3), the modelling and 21 analysis here recommends the use of a mixture for a variety of reasons and, on the balance of resistancemanagement and other considerations for SC1, especially a mixture with a pyrethroid. Firstly, there is a significant advantage of using multiple insecticides in concert. This advantage primarily comes from delaying the evolution of resistance to the first insecticide that breaks, where it can more than double the time that a population broadly remains susceptible to that insecticide. There is also an advantageous effect on the second-to-break in contrast to a non-strategy (sequences), but this effect is around a $10 \%$ delay. Secondly, the advantage of using SC1 in particular alongside another insecticide is likely to be 
1 even more acute because of its unusual property of having a target-site that is encoded in the

2 mitochondrial genome. Were resistance to evolve as a target-site mutation, there is a $\sim 70 \%$ chance that

3 the mitochondrial resistance allele would be the first-to-break, and so using multiple insecticides in

4 concert may be especially important for SC1. This cannot be used to suggest that having a

5 mitochondrially-inherited target-site is undesirable for resistance management because the modelling

6 here only examines the impact of selection in the rate of spread of a pre-existing resistance allele, and

7 excludes the time it may take for a resistance allele to arise in the first place, which is expected to be

8 longer than with a nuclear resistance allele because of haploidy and maternal inheritance; the time to

9 mutation is likely to be especially important for SC1 because a survey of fungicide use in Africa shows

10 a very low historic application of strobilurin chemistries, suggesting that there is minimal to no prior or

11 secondary selection for resistance to this chemistry from agriculture (unpublished data, Bristow \&

12 Firth). Thirdly, despite uncertainties, using SC1 or another insecticide as part of a mixture is likely to

13 be more robust in delaying the evolution of resistance than other strategies. With a new insecticide having to have high effectiveness (>80\%) to meet WHO criteria (World Health Organization, 2001,

15 2012), a mixture tends to outperform other strategies across insecticide effectiveness and female

16 exposure, which represent the key dimensions of partner choice and geographic variability (Figures 4,

17 5, 6). Fourthly, the time to resistance is delayed more by choosing a mixture strategy over other strategies than by choosing one insecticide partner over others. This has relevance to the choice of partner insecticide where there is pre-existing pyrethroid-resistance that reduces pyrethroid effectiveness: Although a mixture of two new insecticides is ideal from the focused-perspective of delaying the evolution of resistance, a mixture of a new insecticide with a pyrethroid (that tends to have lower effectiveness $<80 \%$ ) is preferable to non-mixture strategies with two new insecticides. Fifthly, for a mixture with $\mathrm{SC1}$, a pyrethroid has a major advantage in reducing the overall cost per ITN compared to a new insecticide. As a significant part of the cost of an ITN is the cost of the insecticide and pyrethroids are very cheap compared to new insecticides, the relative time of delayed resistance between partner insecticides with higher or lower effectiveness is marginal compared to the cost difference. A cheaper ITN would presumably enable more ITNs to be procured in a given region. Sixthly, a mixture strategy in the form of a single ITN is immune to deployment error or noncompliance, 
1 which adds ignored complexity to the assessment of rotations and mosaics. The strategies that are being

2 discussed are idealised; deployment error and noncompliance could make real-life rotations and

3 mosaics more like the idealised non-strategy (sequences), which would further increase the relative

4 benefit of mixtures. Lastly, the criteria for WHO approval require ITNs to pass stringent safety and

5 efficacy tests, which for a mixture ITN would require both insecticides to pass safety and efficacy

6 standards independently and together. This adds additional economic cost and development time for

7 mixture ITNs (which is problematic), albeit that these additional costs are lessened with the partner

8 insecticide being a pyrethroid because it has already obtained WHO approval. Therefore, conditional

9 upon the feasibility of its manufacture, the use of SC1 (and other new insecticides) alongside a partner

10 insecticide in a mixture could help to build resistance-management into the bed-net design, but there

11 are significant economic challenges to producing a mixture, such that a pyrethroid may be an attractive

12 choice for its cost-effectiveness. Further work is needed to understand how to balance resistance-

13 management benefits and economic costs to ensure high levels of mosquito control for a sufficiently

14 long time to provide the greatest chance of eradicating malaria.

\section{Conclusions}

17 This study builds on the well-known modelling framework used in Levick et al. (2017) with significant

18 improvements on parameter space explored, the strategies considered, and also on computational

19 resources employed. Here, we find that mixtures tend to be a far superior conceptual resistancemanagement strategy across most of the parameter space. And, when it comes to deploying new

21 insecticides for use in ITNs (e.g. SC1), their durability and impact can be maximised if these are 22 integrated into a mixture product concept (even when the mixture partner is a pyrethroid). The 23 theoretical results presented here can serve as a predictive guideline to bring these new insecticides to market in an evolutionarily robust way - minimising the effects of resistance - and improving the chances of eradicating malaria in the coming decades. 


\section{$1 \quad$ List of abbreviations}

Not applicable.

\section{Availability of data and materials}

22 Data derived from the simulations executed for this study are available at the Zenodo Digital

23 Repository: https://doi.org/10.5281/zenodo.5074771. The source code behind the model is available in

24 GitHub as an $\mathrm{R}$ package called 'detsims': https://github.com/rkanitz/Madgwick-Kanitz-detsims. 
1 Instructions on how to reproduce the simulations and analyse the results are provided in the package's

2 README file, also showed as the main content in the package's GitHub homepage.

3 Competing interests

4 Both authors are employed by Syngenta, a commercial organization involved in research and 5 development.

$6 \quad$ Funding

7 This work was conducted during a postdoctoral research position funded by the Innovative Vector

8 Control Consortium (IVCC).

9 Authors' contributions

10 PGM was responsible for the execution of this work. Both authors designed the research, interpreted

11 its results, and wrote and approved this manuscript.

\section{Acknowledgments}

13 Syngenta gratefully acknowledges the financial support and technical advocacy of the Innovative

14 Vector Control Consortium (IVCC). The computations were performed in Syngenta's scientific computation facilities at the Jealott's Hill research station, supported by Ian Hayhurst, Elisabeth Easton and Steve Cobrin. The authors are thankful to Philip Wege, Andy Bywater, Russell Slater, Dietrich Hermann and Ariane Le Gros for their input on previous versions of this manuscript, and to Ian Hastings and Andy South for early discussions and explanations that contributed to the development of this study.

\section{References}

Bartlett, D.W., Clough, J.M., Godwin, J.R., Hall, A.A., Hamer, M. \& Parr-Dobrzanski, B. 2002. The strobilurin fungicides. Pest Manag. Sci. 58: 649-662.

Bhatt, S., Weiss, D.J., Cameron, E., Bisanzio, D., Mappin, B., Dalrymple, U., et al. 2015. The effect of malaria control on Plasmodium falciparum in Africa between 2000 and 2015. Nature 526: 207-211. 
1 Briët, O.J.T., Penny, M.A., Hardy, D., Awolola, T.S., Van Bortel, W., Corbel, V., et al. 2013. Effects of pyrethroid resistance on the cost effectiveness of a mass distribution of long-lasting insecticidal nets: A modelling study. Malar. J. 12: 1-12.

Charlwood, J.D. 2020. The Ecology of Malaria Vectors. CRC Press.

Churcher, T.S., Lissenden, N., Griffin, J.T., Worrall, E. \& Ranson, H. 2016. The impact of pyrethroid resistance on the efficacy and effectiveness of bednets for malaria control in Africa. Elife 5: 126.

Gould, F. 1986. Simulation Models for Predicting Durability of Insect-resistant Germ Plasm: A Deterministic Diploid, Two-locus Model. Environ. Entomol. 15: 1-10.

Hancock, P., Hendriks, C., Tangena, J.-A., Gibson, H., Hemingway, J., Coleman, M., et al. 2020. Mapping Trends in Insecticide Resistance Phenotypes in African Malaria Vectors. bioRxiv

Hemingway, J., Ranson, H., Magill, A., Kolaczinski, J., Fornadel, C., Gimnig, J., et al. 2016. Averting a malaria disaster: Will insecticide resistance derail malaria control? Lancet 387: 1785-1788.

Hothorn, T., Seibold, H. \& Zeileis, A. 2015. partykit.

Hueter, O.F., Miller, N.A., Wege, P., Hoppé, M.., Maienfisch, P. \& Turnbull, M.D. 2016. Mosquito

Innovative Vector Control Consortium. 2020. Annual Report 2019-2020.

Killeen, G.F. 2020. Control of malaria vectors and management of insecticide resistance through universal coverage with next-generation insecticide-treated nets. Lancet 395: 1394-1400.

Killeen, G.F. \& Ranson, H. 2018. Insecticide-resistant malaria vectors must be tackled. Lancet 391: 1551-1552. The Author(s). Published by Elsevier Ltd. This is an Open Access article under the CC BY 4.0 license.

Kleinschmidt, I., Bradley, J., Knox, T.B., Mnzava, A.P., Kafy, H.T., Mbogo, C., et al. 2018. 
Implications of insecticide resistance for malaria vector control with long-lasting insecticidal nets: a WHO-coordinated, prospective, international, observational cohort study. Lancet Infect. Dis. 18: 640-649.

Levick, B., South, A. \& Hastings, I.M. 2017. A Two-Locus Model of the Evolution of Insecticide Resistance to Inform and Optimise Public Health Insecticide Deployment Strategies. PLoS Comput. Biol. 13: 1-35.

Ranson, H. \& Lissenden, N. 2016. Insecticide Resistance in African Anopheles Mosquitoes: A Worsening Situation that Needs Urgent Action to Maintain Malaria Control. Trends Parasitol. 32: 187-196. Elsevier Ltd.

REX Consortium. 2010. The skill and style to model the evolution of resistance to pesticides and drugs. Evol. Appl. 3: 375-390.

Roll Back Malaria Partnership. 2020. 2 billion mosquito nets delivered worldwide since 2004.

South, A. \& Hastings, I.M. 2018. Insecticide resistance evolution with mixtures and sequences: A model-based explanation. Malar. J. 17: 1-20. BioMed Central.

South, A., Lees, R., Garrod, G., Carson, J., Malone, D. \& Hastings, I. 2019. The role of windows of selection and windows of dominance in the evolution of insecticide resistance in human disease vectors. Evol. Appl. 738-751.

Stone, C. \& Gross, K. 2018. Evolution of host preference in anthropophilic mosquitoes. Malar. J. 17: 1-11. BioMed Central.

Strode, C., Donegan, S., Garner, P., Enayati, A.A. \& Hemingway, J. 2014. The Impact of Pyrethroid Resistance on the Efficacy of Insecticide-Treated Bed Nets against African Anopheline Mosquitoes: Systematic Review and Meta-Analysis. PLoS Med. 11.

Takken, W., Costantini, C., Dolo, G., Hassanali, A., Sagnon, N. \& Osir, E. 2006. Mosquito mating behaviour. Bridg. Lab. F. Res. Genet. Control Dis. Vectors 183-188.

Waite, J.L., Swain, S., Lynch, P.A., Sharma, S.K., Haque, M.A., Montgomery, J., et al. 2017. 

Increasing the potential for malaria elimination by targeting zoophilic vectors. Sci. Rep. 7: 1-10. Nature Publishing Group.

3 Wege, P., Hueter, O., Hoppé, M., Maienfisch, P., Nourani, R. \& Daniels, M. 2021. Discovery and optimization of novel mosquitocidal compounds for the control of anopheline vectors of malaria. In: Recent Highlights in the Discovery and Optimization of Crop Protection Products, pp. 1-13.

Wilson, A.L., Courtenay, O., Kelly-Hope, L.A., Scott, T.W., Takken, W., Torr, S.J., et al. 2020. The importance of vector control for the control and elimination of vector-borne diseases.

World Health Organization. 2012. Global Plan for Insecticide Resistance Management in Malaria Vectors.

World Health Organization. 2001. Guidelines for laboratory and field-testing of long-lasting insecticidal nets.

12 World Health Organization. 2020a. Prequalification Vector Control.

13 World Health Organization. 2013. Report of the Sixteenth WHOPES Working Group Meeting.

14 World Health Organization. 2017. Report of the Twentieth WHOPES Working Group Meeting.

15 World Health Organization. 2019. World Malaria Report 2019.

16 World Health Organization. 2020b. World Malaria Report 2020.

17 Yukich, J.O. \& Chitnis, N. 2017. Modelling the implications of stopping vector control for malaria control and elimination. Malar. J. 16: 1-16. BioMed Central. 


\section{Supplementary Files}

This is a list of supplementary files associated with this preprint. Click to download.

- FigS178.pdf

- SupplementaryFigureLegends.docx 